\title{
Bypassing the central bottleneck after single-task practice in the psychological refractory period paradigm: Evidence for task automatization and greedy resource recruitment
}

\author{
François Maquestiaux \\ Université Paris-Sud, Orsay, France \\ Maude Laguë-Beauvais \\ Université du Québec à Montréal et Institut Universitaire de Gériatrie de Montréal, \\ Montréal, Québec, Canada \\ ERIC RUTHRUfF \\ University of New Mexico, Albuquerque, New Mexico \\ AND \\ LOUIS BHERER \\ Université du Québec à Montréal et Institut Universitaire de Gériatrie de Montréal, \\ Montréal, Québec, Canada
}

\begin{abstract}
In this research, the controversial issue of whether the central bottleneck can be bypassed through task automatization was investigated. To examine this issue, participants received six single-task practice sessions with an auditory-vocal task (low vs. high pitch). We then assessed dual-task performance using the analytically tractable psychological refractory period (PRP) paradigm, in which the highly practiced auditory-vocal task was presented as Task 2 , along with an unpracticed visual-manual Task 1 . The results provide evidence of bottleneck bypass for virtually all the participants (17 out of 20 ). Several converging tests suggest that the bottleneck reemerged, however, in a follow-up experiment with tasks presented in the opposite order (auditory-vocal Task 1 and visual-manual Task 2). One possible explanation is that tasks greedily recruit central resources when available, even though they can operate without central resources when unavailable.
\end{abstract}

Laboratory experiments using novel tasks have consistently shown that humans have great difficulty performing two cognitive tasks at once (see Pashler, 1998). Similar problems have also been documented in real-world settings, with more practiced tasks. For instance, cell phone conversations disrupt driving performance, even with hands-free cell phones (e.g., Strayer, Drews, \& Johnston, 2003). Furthermore, Levy, Pashler, and Boer (2006) found that even a well-practiced vehicle-braking task was subject to interference when performed concurrently with a speeded choice response task (see also Van Selst, Ruthruff, \& Johnston, 1999).

For many decades, cognitive psychologists have sought to understand this dual-task interference, because of its practical and theoretical significance. The study of dualtask interference and of ways to reduce it are of great practical significance for populations such as drivers, aircraft pilots, and air traffic controllers. It is also of great theoreti- cal importance to those seeking to understand the processing limitations inherent to human cognitive architecture.

Because it is particularly tractable, the dual-task paradigm used most widely (including in the present experiments) is the psychological refractory period (PRP) procedure. This procedure involves the presentation of two distinct stimuli (S1 and $\mathrm{S} 2$ ), each requiring a distinct overt speeded response (R1 and R2). The interval between the onset of $\mathrm{S} 1$ and $\mathrm{S} 2$, called the stimulus onset asynchrony (SOA), is manipulated. At long SOAs (about 1,000 msec), there is little or no temporal overlap between the performance on Task 1 and Task 2. Thus, long SOAs provide a baseline measure of response time (RT) for the tasks performed independently. At short SOAs, there is considerable temporal overlap between Task 1 and Task 2. Typically, the instructions encourage participants to respond as quickly and accurately as possible to each task, while emphasizing the speed of Task 1 responses. 
The classical finding in PRP experiments is Task 2 RT (RT2) lengthening of several hundreds of milliseconds as SOA decreases, whereas Task 1 RT (RT1) remains relatively unaffected. First observed by Telford (1931), this RT2 lengthening with decreasing SOA has been termed the PRP effect (for reviews, see Lien \& Proctor, 2002; Meyer \& Kieras, 1997b; Pashler, 1994a). The fact that the PRP effect is usually concentrated on Task 2 alone (a consequence of the emphasis on Task 1 performance) makes it particularly easy to derive predictions from candidate models. The PRP effect is also remarkably robust across a wide range of experimental conditions, occurring even with simple tasks that do not utilize the same input or output systems.

\section{Theories of the PRP Effect}

Welford (1952) proposed that the PRP effect is due to an inability to carry out more than one central mental operation (e.g., response selection) at once. This proposal, known as the central bottleneck model, is depicted in Figure 1. Each task is modeled as three successive processing stages: the precentral stage (A), central stage (B), and postcentral stage $(\mathrm{C})$. A preliminary hypothesis is that precentral stages (e.g., stimulus identification) and postcentral stages (e.g., response execution) of one task can be carried out in parallel with any stage of the other task. The central stages, however, are constrained by a processing bottleneck, preventing Stage 2B from being carried out while Stage 1B is still underway. At short SOAs, this bottleneck results in a waiting period called the bottleneck delay (represented by the horizontal dashed line in Figure 1). The bottleneck delay causes the PRP effect, which can be expressed in terms of the durations of the component stages of Task 1 and Task 2 (Pashler \& Johnston, 1989; Ruthruff, Johnston, \& Van Selst, 2001): ${ }^{1}$

$$
\mathrm{PRP} \text { effect }=1 \mathrm{~A}+1 \mathrm{~B}-2 \mathrm{~A}-\mathrm{SOA}_{\text {short }} .
$$

Because the PRP equation given above does not contain terms for Stages 2B and 2C, it follows that the PRP effect is not influenced by the duration of the Task 2 central and postcentral stages. Accordingly, increasing the duration of Task 2 processing stages at or after the Task 2 central stage will increase Task 2 processing time by a fixed amount, whatever the SOA. This prediction has been termed the Task 2 additivity prediction. In contrast, because the PRP effect equation contains terms for Stages 1A and 1B, it follows that the bottleneck delay is influenced by the duration of the Task 1 precentral and/ or central stages. Accordingly, increases in the duration of Task 1 processing stages up to and including the central stage should carry over fully onto RT2 at short SOAs (where the bottleneck occurs), but not at long SOAs. In other words, the effect of Task 1 difficulty on RT2 should be large at short SOAs (where carryover occurs) and small at long SOAs (where carryover should not occur). This prediction has been termed the Task 1 carryover prediction. These predictions, along with others, have been verified several times (e.g., McCann \& Johnston, 1992; Pashler \& Johnston, 1989).
Also, because RT1 $=1 \mathrm{~A}+1 \mathrm{~B}+1 \mathrm{C}$, the bottleneck model equation can be rewritten as

$$
\mathrm{PRP} \text { effect }=\mathrm{RT} 1-1 \mathrm{C}-2 \mathrm{~A}-\mathrm{SOA}_{\text {short }} .
$$

From this equation and the assumption that the duration of input and output stages are stable across practice, it follows that a plot of the size of the PRP effect and RT1 should show a linear relation with a slope of 1.0. This prediction was confirmed by Van Selst et al. (1999) and by Ruthruff, Johnston, and Van Selst (2001). For the usual PRP tasks, such as pressing a button in response to a letter, it seems reasonable that noncentral stages (i.e., identifying letters and pushing buttons) should be relatively stable across practice (see Van Selst et al., 1999). However, if these stages do decrease with practice, the result would be a slope of less than 1 . This prediction is consistent with data from younger and older adults reported by Maquestiaux, Hartley, and Bertsch (2004).

Although it is generally accepted that a central bottleneck occurs in PRP experiments, different accounts for the bottleneck exist. Meyer and Kieras (1997a, 1997b) argued that the central bottleneck often reflects a strategic deferment of the Task 2 central stage while the Task 1 central stage is still underway (see also Schumacher et al., 1999). In this view, the central stages of Task 1 and Task 2 can proceed either in sequence or in parallel, depending on the experimental demands (e.g., successive presentation vs. simultaneous presentation of the two imperative stimuli)

\section{Time Course for Task 1 and Task 2 at Short SOAs}

S1

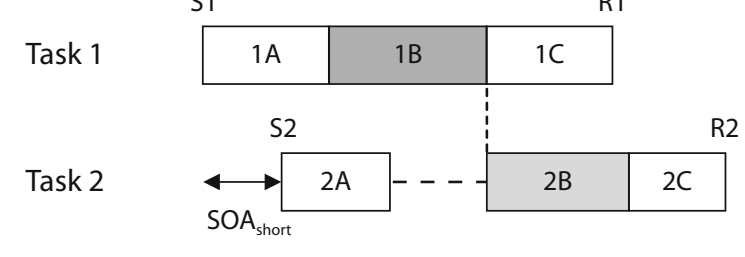

Time Course for Task 1 and Task 2 at Long SOAs

S1

Task 1

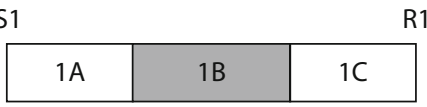

S2

$\mathrm{R} 2$

Task 2

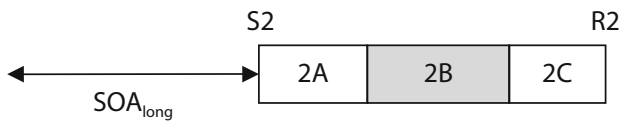

Figure 1. Central bottleneck model. $R 1$ and $R 2$ represent the Task 1 and Task 2 responses to the stimuli S1 and S2, which are separated by the stimulus onset asynchrony (SOA). Each task is divided into three distinct processing stages: the precentral stage (A), the central stage (B), and the postcentral stage (C). The precentral and postcentral stages of one task are assumed to operate in parallel with all stages of the other task. At short SOAs, the Task 2 central stage must wait until the Task 1 central stage has finished, resulting in a waiting period called bottleneck delay (represented by the horizontal dashed line). At long SOAs, Task 2 does not need to wait, because there is no temporal overlap in the demand for the Task 1 and Task 2 central stages. 
and the instructions (e.g., emphasize Task 1 processing speed vs. weighting both tasks equally). Despite the lack of a universal agreement on whether the central bottleneck reflects a structural limitation or a strategic postponement, attempts to encourage bottleneck bypassing at low levels of practice have failed (Hartley \& Maquestiaux, 2007; Levy \& Pashler, 2001; Lien, McCann, Ruthruff, \& Proctor, 2005; Pashler, 1994a, 1994b; Ruthruff, Pashler, \& Hazeltine, 2003; Ruthruff, Pashler, \& Klaassen, 2001).

\section{Does Practice Reduce, or Even Eliminate, the PRP Effect?}

Initial attempts to study the effects of practice on PRP performance produced a surprisingly modest reduction in the size of the PRP effect (e.g., Bertelson \& Tisseyre, 1969; Borger, 1963; Karlin \& Kestenbaum, 1968). For instance, Bertelson and Tisseyre obtained large mean PRP effects of roughly 195 and $260 \mathrm{msec}$ following four and seven practice sessions in Experiments 1 and 2, respectively. At first glance, it appears that the cognitive limitations underlying the PRP effect are resistant to practice. But this conclusion is suspect, given that these studies employed pairs of tasks requiring manual responses. Research suggests that independent control of the two hands is difficult, even impossible (e.g., De Jong, 1993; Keele, 1973; Meyer $\&$ Kieras, 1997b). For instance, De Jong suggested that manual responses generate strong output conflicts, due to a bottleneck localized at the level of response generation.

Van Selst et al. (1999) suspected that the use of manual responses is responsible for the apparent resistance of PRP interference to practice. Therefore, they conducted a study with two distinct response modalities: vocal (Task 1) and manual (Task 2). They observed large PRP reductions after 18 practice sessions: The PRP dropped from $353 \mathrm{msec}$ in the 1 st session to only $40 \mathrm{msec}$ in the 18 th session. Despite the dramatic PRP reduction, several predictions derived from the central bottleneck model were confirmed late in practice, suggesting that performance was still limited by a central bottleneck.

Ruthruff, Johnston, Van Selst, Whitsell, and Remington (2003) brought back to the laboratory 1 participant from the Van Selst et al. (1999) study who had produced no observable dual-task interference. The goal was to determine whether the central bottleneck was truly bypassed or was just latent. Bypassing the central bottleneck refers to the parallel processing of the Task 1 and Task 2 central stages, without necessarily eliminating the Task 1 central stage and/or the Task 2 central stage. In contrast, a latent bottleneck refers to a lack of observable interference (despite an intact central bottleneck), because the central stages of Task 1 and Task 2 are rarely demanded at the same time. Preliminary results replicated the absence of PRP interference observed in Van Selst et al. for this participant. Then, to increase the likelihood of temporal overlap between Task 1 and Task 2 central operations, Ruthruff, Johnston, et al. presented Task 2 earlier in time (i.e., reduced all of the SOAs by $183 \mathrm{msec}$ ). This manipulation led to the emergence of a PRP effect: Mean RT2 was longer by $30 \mathrm{msec}$ at the $-216-\mathrm{msec} \mathrm{SOA}$, relative to the $617-\mathrm{msec}$ SOA. The authors concluded that the absence of the PRP effect for this participant in Van Selst et al. was very likely due to an absence of temporal alignment between the demands for the Task 1 and Task 2 central operations, rather than to an elimination of the limitation underlying the central bottleneck. These results demonstrate that the absence of PRP interference does not necessarily indicate the absence of a processing bottleneck (see also Lien, Ruthruff, \& Johnston, 2006).

Recent experiments using simultaneous dual-task presentation (0-msec SOAs) have produced very little dualtask interference after practice (Hazeltine, Teague, \& Ivry, 2002; Schumacher et al., 2001). Schumacher et al. (2001) claimed that, after practice, participants achieved "virtually perfect time sharing in the dual-task performance of basic choice reaction tasks" (p. 101). But because RTs were of relatively short duration (less than or equal to $300 \mathrm{msec}$ ), it is difficult, or even impossible, to determine whether the central bottleneck was bypassed or just latent (Ruthruff, Johnston, et al., 2003). Hazeltine et al. (2002) explicitly acknowledged this possibility. Also, Anderson, Taatgen, and Byrne (2005) were able to simulate the nearperfect time sharing obtained by Hazeltine et al. (2002) with a model incorporating a central bottleneck.

In addition to the latent bottleneck issue, Tombu and Jolicœur (2004) pointed out several methodological flaws in Schumacher et al.'s (2001) study that may have obscured dual-task interference. The flaws include differences in the amount of mobilized effort between conditions, trial type uncertainty, omission of an expected stimulus, and task-switching costs present for single- and dual-task trials in mixed blocks but absent for single-task trials in pure blocks. Using experimental designs that greatly reduced these technical problems, they found robust dual-task costs, challenging Schumacher et al.'s (2001) claim of virtually perfect time sharing.

Ruthruff, Van Selst, Johnston, and Remington (2006), arguably, provided more convincing evidence of bottleneck bypass (under some conditions). Participants first received one of three training types for eight sessions: singletask training on Task 1, single-task training on Task 2, or dual-task training on both Task 1 and Task 2 . Then all the participants performed four dual-task test sessions. In Experiment 1 , Task 1 was a speeded choice response task requiring a vocal response to a tone, and Task 2 was a speeded choice response task requiring a manual response to an alphanumeric character. Experiment 1's results were consistent with an intact central bottleneck, whose duration decreased as Task 1 practice increased.

Experiment 2 replicated Experiment 1 with the reverse task order; the auditory task became Task 2 and the visual task became Task 1 . The results were consistent with an intact central bottleneck for the majority of the participants (14 out of 18). However, several converging indicators demonstrated that 4 participants had actually eliminated the bottleneck after practice. First, these 4 participants reversed the response order (R2 before R1) on a very high proportion of trials (from $66 \%$ to $98 \%$ ) at the shortest SOA. According to the central bottleneck model, $\mathrm{R} 1$ should almost always be emitted before R2 because Task 1 central operations are assumed to be performed 
before Task 2 central operations. But either response order is possible when the bottleneck has been bypassed and central stages are performed simultaneously. Second, these 4 participants with high frequencies of response reversals also happened to produce unusually small PRP effects (from 33 to $67 \mathrm{msec}$ ), relative to the remaining 14 participants (from 125 to $505 \mathrm{msec}$ ). Third, the effect of Task 1 stimulus-response (S-R) compatibility on RT1 $(62 \mathrm{msec})$ did not carry over fully to RT2 $(13 \mathrm{msec})$, contrary to the Task 1 carryover prediction of the central bottleneck model.

The demonstration of bottleneck bypass by Ruthruff, Van Selst, et al. (2006, Experiment 2) is especially impressive because RT1s were relatively long (from 528 to $609 \mathrm{msec}$ ). In fact, 2 of the participants bypassed the bottleneck even though they did not practice Task 1 in the training phase (a fact that we will take advantage of in the present study). Given the long RT1s and the wide range of SOAs $(17,67,150,250,450$, and $850 \mathrm{msec})$, the absence of interference cannot easily be attributed to a latent bottleneck. It seems highly likely that central operations were often demanded at the same time, at least for some of the SOAs. Although the 4 participants did not completely eliminate PRP interference, the modest residual effects could have been due to some other sources of interference (see, e.g., Hazeltine \& Ruthruff, 2006; Hazeltine, Ruthruff, \& Remington, 2006; Ruthruff, Hazeltine, \& Remington, 2006).

Ruthruff, Van Selst, et al. (2006) identified several factors that might have led to the unusual elimination of the central bottleneck: The use of considerable practice $(4,480$ training trials, followed by 2,240 dual-task test trials), the absence of input modality conflicts and output modality conflicts, and the use of favorable input/output modality pairings (Ruthruff, Hazeltine, \& Remington, 2006; Stelzel, Schumacher, Schubert, \& D'Esposito, 2006). But none of these factors can fully explain the elimination of the bottleneck, because they were present for all the participants in all the conditions in both experiments.

One factor that appears to have been crucial was the task order. In Experiment 1 (Auditory Task 1 and Visual Task 2), none of the 18 participants showed signs of having bypassed the bottleneck. In Experiment 2 (Visual Task 1 and Auditory Task 2), 4 participants out of 18 bypassed the bottleneck. This result was not predicted in advance by the candidate models under consideration, so a post hoc explanation was needed. Ruthruff, Van Selst, et al. (2006) proposed the greedy resource recruitment hypothesis. One assumption was that the auditory task was more amenable to automatization than was the visual task because it was easier (producing shorter RTs). Another assumption was that even automatized tasks that do not need central resources will (greedily) recruit those resources when available. The automatic assignment of central resources, when available, may seem counterintuitive; however, a benefit of this strategy is to relieve the information processing system of the burden of assessing resource demands.

If (as was hypothesized for Experiment 1 in Ruthruff, Van Selst, et al., 2006) an automatized task is performed first, appropriating available central resources, a non- automatized task presented subsequently will experience a bottleneck delay. However, if (as was hypothesized for Experiment 2 in Ruthruff, Van Selst, et al., 2006) the nonautomatized task is performed first, engaging central resources, an automatized task presented subsequently can proceed without delay. This combination of assumptions provides a parsimonious account, albeit post hoc, of the task order effects on the presence/absence of the central bottleneck across experiments in their study.

\section{Goals of the Present Study}

One limitation of the Ruthruff, Van Selst, et al. (2006) study is that only a small percentage of participants bypassed the bottleneck. If bottleneck bypassing is a genuine phenomenon, it should be possible to find more favorable conditions in which virtually all participants would bypass the bottleneck. Experiment 1 was an attempt to find such conditions. To this end, we optimized the procedure used by Ruthruff, Van Selst, et al. (Experiment 2). One major change, motivated by the hypothesis that bottleneck bypassing is due to automatization of the tone task, was to make this task even easier: We used two possible tone pitches, rather than four. Another change was to further increase the number of training trials (5,040 vs. 4,480 trials). A third major difference was the use of only one type of training for all 20 participants: Auditory Task 2 training. Indeed, pairing the highly practiced Task 2 with an unpracticed Task 1 (thereby producing a long mean RT1) is the least likely dual-task situation to produce a latent bottleneck. In other words, evidence of a small PRP effect in this training condition would provide the strongest evidence for bottleneck bypass. Fourth, to facilitate a more accurate assessment of the proportion of participants bypassing the bottleneck, we greatly increased the sample size in the Task 2 training condition from 6 participants to 20 participants.

Looking ahead to the results of Experiment 1, the majority of participants appeared to have bypassed the bottleneck. The goal of the present Experiment 2, therefore, was to test the greedy resource recruitment hypothesis proposed a posteriori by Ruthruff, Van Selst, et al. (2006) to explain the presence of the central bottleneck with one task order, but not with the reversed task order. We simply transferred participants who showed clear evidence that they had bypassed the central bottleneck with a specific task order (Experiment 1) to a dual-task design with the opposite task order (Experiment 2). The greedy resource recruitment hypothesis predicts that, despite even greater amounts of practice on the component tasks, the participants would no longer bypass the bottleneck. The reason is that the automatized tone task would come first, grabbing up resources that would be needed to perform the nonautomatized Task 2.

\section{EXPERIMENT 1}

Following Ruthruff, Van Selst, et al. (2006), Task 1 required a speeded manual keypress (four possible keypresses) to an alphanumeric character (eight possible stimuli). The S-R compatibility of Task 1 was manipulated within sub- 
jects and within blocks. Task 2 was a speeded tone pitch classification (two choices) task requiring a vocal response.

If the bottleneck remains intact with practice and participants perform Task 1 central stages before Task 2 central stages, PRP effects should be fairly large (roughly 200-500 msec). In addition, the effect of the Task $1 \mathrm{com}-$ patibility manipulation on Task 1 RTs should carry over fully to Task 2 RTs at short SOAs, but not at long SOAs.

But if Task 2 practice allows bottleneck bypassing, PRP effects should be very small, and there should be little or no carryover of Task 1 compatibility manipulation to Task 2 RTs at short SOAs (assuming that the participants do not group responses). In addition, the participants might actually respond to the tasks in an order opposite to their presentation order (i.e., R2 before R1) at short SOAs. That is, because Task 2 was easier, it should usually win a parallel race with Task 1.

\section{Method}

Twenty participants performed nine sessions spread over 9 different testing days (three sessions per week). The first phase consisted of six training sessions with Auditory Task 2 only; the second phase consisted of three dual-task sessions.

\section{Participants}

Twenty young adults $(M=24.6$ years, $S D=2.5$ years, range $=$ 20-31 years; 10 women) were recruited from the Université du Québec à Montréal and the Institut Universitaire de Gériatrie de Montréal and were paid (\$10 Canadian per session) to participate in this experiment. They were generally highly educated $(M=17.6$ years, $S D=1.6$ years $)$. On a 5 -point health rating scale $(5=$ excellent health $)$, they gave mean self-ratings of $4.6(S D=0.5)$. They were screened for normal or corrected-to-normal vision and hearing, using self-report. None of the participants reported any difficulties in discriminating the auditory and visual stimuli presented in the experiment.

\section{Stimuli}

Visual Task 1. Task 1 was to identify a single alphanumeric character drawn from the set 1, 2, 3, 4, A, B, C, D, presented in Times New Roman font. The characters subtended approximately $1.49^{\circ}$ vertically by $1.04^{\circ}$ horizontally at a viewing distance of $46 \mathrm{~cm}$. The background was white, and the characters were black.

Auditory Task 2. Task 2 was to identify one of two possible tones presented for $150 \mathrm{msec}$ over headphones. The tone highest in pitch $(1800 \mathrm{~Hz})$ was labeled as a high tone, and the tone lowest in pitch $(400 \mathrm{~Hz})$ was labeled as a low tone.

\section{Apparatus}

Stimulus presentation and timing were performed by a PCcompatible computer equipped with Chant Speechkit Version 4 for detecting speech onset and a Voice Connexion system (Microsoft Speech SDK Version 5.1) for automatically recognizing speech.

\section{Procedure}

The participants responded to the character by pressing the "f," "t," "y," or " $j$ " key on a qwerty keyboard, using the fingers of the right hand (with the exception of the thumb). For half of the participants, the numbers were mapped compatibly $(1,2,3,4)$ onto the four response keys from left to right, whereas the letters were mapped incompatibly (C, A, D, B) onto the same four keys. For the other half of the participants, the letters were mapped in alphabetic order (A, B, C, D) but the numbers were mapped in a scrambled order $(3,1,4,2)$. The participants responded to the pitch of the tone with a vocal response, either "high" or "low" (in French: "haut" or "bas"), into the headset microphone.
During Phase 1, all the participants performed six single-task training sessions with Auditory Task 2, each session consisting of 840 experimental trials. Each training session was broken into 14 blocks of 60 trials, separated by 2-min breaks. During each break, the computer provided feedback on the average speed and average accuracy for the previous block. The participants were instructed to respond as quickly and accurately as possible, as well as to improve their performance from one block to another.

During Phase 2, all the participants performed three dual-task (PRP) test sessions, pairing an unpracticed Visual Task 1 with the highly practiced Auditory Task 2. Because the participants had to learn a new task and a new paradigm, the first test session was considered practice and, therefore, was not included in the dual-task data analysis. Each dualtask session consisted of 20 warm-up dual-task trials followed by 384 experimental dual-task trials. The experimental trials were a random ordering of eight repetitions of the 48 trial types produced by a complete factorial cross of SOA $(15,65,150,250,550$, and 1,000 msec), $\mathrm{S}-\mathrm{R}$ compatibility of Task 1 (compatible or incompatible), and Task 1 response finger (first through fourth finger). All variables were manipulated within blocks. The session was broken into eight blocks of 48 trials, separated by 2-min breaks. During each break, the computer provided feedback on the average speed for Task 1 and the accuracy for both Task 1 and Task 2 in the previous block. The participants were given typical PRP instructions: to respond as quickly and accurately as possible to each task while emphasizing the speed of Task 1 responses. None of them received explicit verbal instructions regarding response order or response grouping.

Each trial began with presentation of a black asterisk for $500 \mathrm{msec}$ in the center of the screen. Then, a foreperiod, varying randomly in duration from 100 to $250 \mathrm{msec}$ (in steps of $50 \mathrm{msec}$ ), was introduced. In the dual-task condition, the Task 1 character appeared in the center of the screen, and the Task 2 tone was played after a variable SOA $(15,65,150,250,550$, or $1,000 \mathrm{msec})$. The Task 1 character remained until a response was registered or $2,500 \mathrm{msec}$ had elapsed. In the Task 2 training condition, only the tone was presented. The timing of the Task 2 tone in this single-task condition was yoked to that in the dual-task condition: Following the random foreperiod, there was an additional "SOA" delay.

After each trial, a message displayed for $600 \mathrm{msec}$ informed the participants whether they had made an erroneous or a correct response on the two tasks in the dual-task condition or on Task 2 in the single-task condition. Also, if the participants responded to a stimulus within $100 \mathrm{msec}$ of its onset, a "too early" (in French: "trop rapide") message was displayed for $600 \mathrm{msec}$. If the participants failed to respond to a stimulus within $2,500 \mathrm{msec}$ of its onset, a "tooslow" message (in French: "trop lent") was displayed for $600 \mathrm{msec}$. The intertrial interval was $1,000 \mathrm{msec}$.

\section{Analyses}

For the Auditory Task 2 training sessions, we conducted separate ANOVAs on mean RT2 and Task 2 error rate, using session (1, 2, 3, 4,5 , and 6) as a within-subjects variable. In the dual-task phase (Sessions 8 and 9), we conducted separate ANOVAs on mean RT1, RT2, response reversal rate (computed as the percentage of trials on which a Task 2 response was emitted before the Task 1 response), Task 1 error rate, and Task 2 error rate, using the factors of SOA and Task 1 S-R compatibility (compatible vs. incompatible) as within-subjects variables. Only single-task trials with correct responses and latencies between 100 and 2,500 msec were included in the RT analysis. Only dual-task trials with correct responses and latencies between 100 and 2,500 $\mathrm{msec}$ on both Task 1 and Task 2 were included in the RT analysis. Application of the RT cutoffs led to the removal of $3.29 \%$ and $4.55 \%$ of the trials in the single-task and dual-task conditions, respectively.

\section{Results}

The Results section contains two sets of statistical analyses. We first will present the results from all 20 participants. 
Looking ahead, the overall analysis strongly hinted at categorical performance differences among participants. Consequently, a second set of analyses was carried out, one for each of the three suspected subgroups of participants (i.e., bottleneckers, bypassers, and bypass groupers).

\section{Training Phase}

\section{Analysis of All Participants}

Figure 2 shows the decline in RT2 across the six training sessions. Task 2 training reduced RT2 by $36 \%$, from $478 \mathrm{msec}(S D=90 \mathrm{msec})$ in Session 1 to $308 \mathrm{msec}(S D=$ $57 \mathrm{msec})$ in Session $6[F(5,95)=72.65, p<.001]$. The average Task 2 error rate was $4.95 \%$. The overall Task 2 error rates were $6.1 \%, 6.1 \%, 5.2 \%, 3.2 \%, 4.0 \%$, and $5.1 \%$ in Sessions 1, 2, 3, 4, 5, and 6, respectively; this effect did not reach statistical significance $[F(5,95)=1.16, p=.33]$.

\section{Visual Task 1 RTs in the Test Sessions}

Figure 3 shows mean RT1 and RT2 as a function of SOA, averaged across the last two test sessions (i.e., Sessions 8 and 9). There was a significant main effect of SOA on $\mathrm{RT} 1[F(5,95)=2.61, p<.03]$. Post hoc comparisons using the Bonferroni procedure showed that RT1 was significantly longer (by $29 \mathrm{msec}$ ) at the 1,000-msec SOA $(M=665 \mathrm{msec}, S D=130 \mathrm{msec})$ than at the $250-\mathrm{msec}$ SOA $(M=636 \mathrm{msec}, S D=107 \mathrm{msec})$; no other comparison was significant. The Task $1 \mathrm{~S}-\mathrm{R}$ compatibility effect was $172 \mathrm{msec}$, with a longer mean RT1 in the incompatible condition $(M=734 \mathrm{msec})$ than in the compatible condition $(M=562 \mathrm{msec})[F(1,19)=112.22, p<$ $.001]$. SOA and Task $1 \mathrm{~S}-\mathrm{R}$ compatibility did not interact $[F(5,95)<1]$.

\section{PRP Effect on Auditory Task 2 \\ in the Test Sessions}

The PRP effect was computed as the difference between RT2 at the $15-\mathrm{msec} \mathrm{SOA}(M=588 \mathrm{msec}, S D=214 \mathrm{msec})$ and the $1,000-\mathrm{msec} \operatorname{SOA}(M=372 \mathrm{msec}, S D=93 \mathrm{msec})$. The mean PRP effect was $216 \mathrm{msec}$.

\section{PRP Effect Versus RT1 for Individuals}

Figure 4 is a plot of the mean PRP effect during the test sessions as a function of mean RT1 for each participant. There was enormous variation in the size of the PRP effect across participants, hinting at categorical differences in how they performed the tasks. The size of the PRP effect was large $(>400 \mathrm{msec})$ for a few participants but fell below $100 \mathrm{msec}$ for 6 participants.

Assuming that the central bottleneck remained intact and that the participants differ mainly in their central stage durations, there should be a linear relationship between the PRP effect and RT1 across participants with a slope of 1 (Ruthruff, Johnston, \& Van Selst, 2001; Van Selst et al., 1999). In contrast with the bottleneck model prediction, the linear fit was very weak $\left(r^{2}=.046\right)$, and the overall slope was only .324. To rescue the bottleneck model, it could be proposed that the participants varied dramatically in the duration of their noncentral stages. It seems unlikely, however, that highly practiced noncentral stages, such as identifying a tone or pushing a button, would vary so dramatically across participants (a few hundred milliseconds).

\section{Task 1 Carryover Prediction}

The bottleneck model predicts that the effects of increasing the duration of Task 1 processing stages up to and including the central stage should carry over fully to RT2 at short SOAs, but not at long SOAs. This prediction was tested using the Task $1 \mathrm{~S}-\mathrm{R}$ compatibility manipulation, which had a very large effect on Task 1 itself $(172 \mathrm{msec})$. Figure 5 shows how Task $1 \mathrm{~S}-\mathrm{R}$ compatibility influenced RT1 (horizontal dashed line) and RT2 (filled symbol) at each SOA. The effect of Task $1 \mathrm{~S}-\mathrm{R}$ compatibility carried over to RT2 more at the 15-msec SOA (mean RT2 difference between the incompatible and the compatible conditions was $68 \mathrm{msec}$ ) than at the 1,000-msec SOA $(23 \mathrm{msec})$, leading to a significant interaction of Task 1 S-R compatibility and SOA $[F(5,95)=8.71, p<.001]$. However, the carryover to RT2 at the shortest SOA was only $39.5 \%$ of the effect on RT1 and was significantly

Task 2 Response Time Across Training Sessions

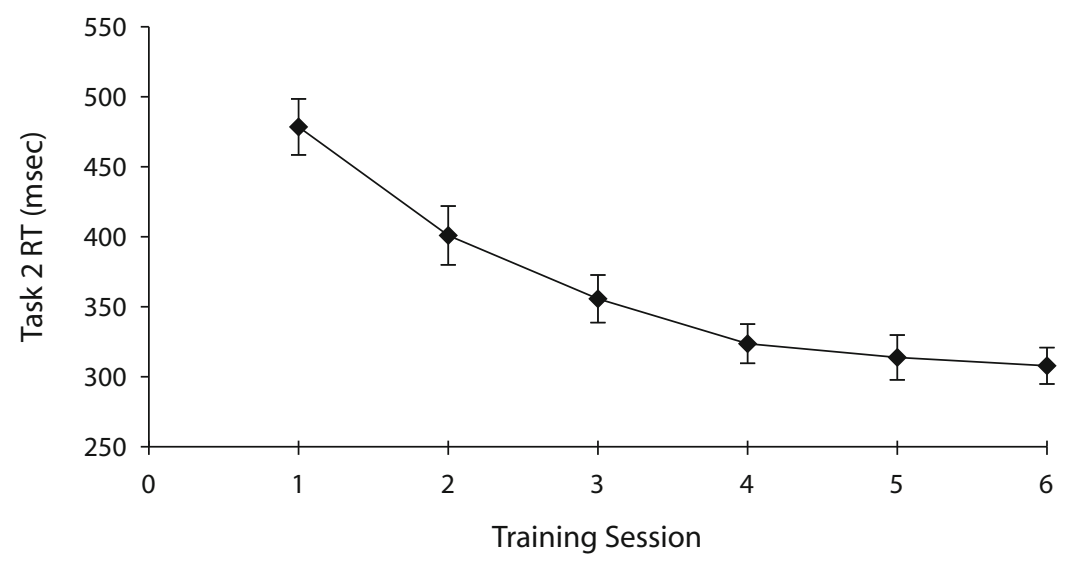

Figure 2. Mean Task 2 response times (RTs) in Experiment 1 as a function of training session. Bars show standard errors (calculated on the basis of between-subjects variance in the mean for each condition). 

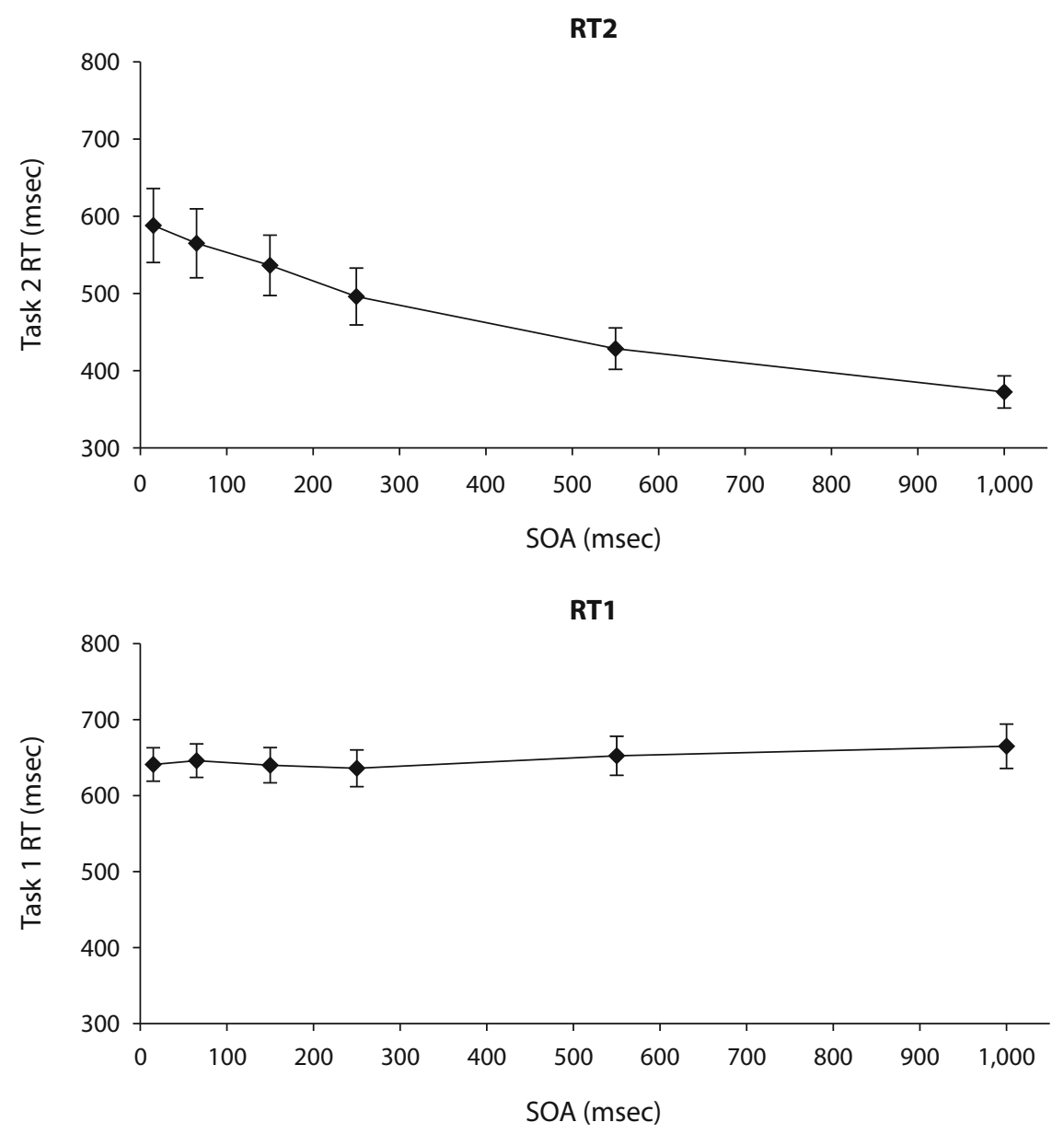

Figure 3. Mean Task 1 and Task 2 response times (RTs) in Experiment 1 as a function of stimulus onset asynchrony (SOA). Bars show standard errors.

smaller than the $100 \%$ carryover predicted by the central bottleneck model $[t(19)=5.01, p<.001]$.

\section{Response Reversal Rate}

According to the bottleneck model, central processing must occur in a sequential order. It is usually assumed that Task 1 central processing will precede Task 2 central processing, due to S1 always preceding S2 and to instructions prioritizing Task 1. Consequently, responses should be emitted in the same sequential order (Task 1 response followed by Task 2 response) whatever the SOA. That is, the response reversal rate should be close to $0 \%$, as is almost always the case in PRP studies. Figure 6 shows the mean response reversal rate as a function of SOA and Task 1 S-R compatibility, averaged across the last two sessions (i.e., Sessions 8 and 9). Inconsistent with the standard bottleneck model, response reversals were frequent $(27.6 \%)$. The overall response reversal rate was larger in the incompatible condition $(M=33.1 \%)$ than in the compatible condition $(M=22.0 \%)[F(1,19)=$ $41.38, p<.001]$. In addition, there was a main effect of SOA $[F(5,95)=45.51, p<.001]$, reflecting increasing response reversal rates with decreasing SOAs; the mean response reversal rate was $56.3 \%$ at the 15 -msec SOA but only $0.7 \%$ at the $1,000-\mathrm{msec}$ SOA. There was also a twoway interaction between SOA and Task $1 \mathrm{~S}-\mathrm{R}$ compatibility $[F(5,95)=12.30, p<.001]$, because the participants were most likely to emit R2 before R1 if the SOA was short and Task 1 was incompatible. These are exactly the conditions in which Task 2 has the best chance to win a parallel race with Task 1.

\section{Task 1 and Task 2 Error Rates}

Visual Task 1. The participants made more errors on Task 1 in the incompatible S-R condition $(8.9 \%)$ than in the compatible S-R condition $(3.3 \%)[F(1,19)=$ $46.07, p<.001]$. There was also a main effect of SOA $[F(5,95)=6.08, p<.001]$, reflecting a tendency to make more Task 1 errors at short SOAs, relative to long SOAs; Task 1 error rates were $8.0 \%, 6.7 \%, 6.6 \%, 6.0 \%$, $4.9 \%$, and $4.2 \%$ at the $15-, 65-, 150-, 250-, 550-$, and 1,000 -msec SOAs, respectively. There was no two-way interaction between SOA and Task $1 \mathrm{~S}-\mathrm{R}$ compatibility $[F(5,95)=1.07, p=.38]$.

Auditory Task 2. Task $1 \mathrm{~S}-\mathrm{R}$ compatibility did not affect Task 2 error rates $[F(1,19)=1.33, p=.26]$. There was, 
PRP vs. RT1 by Participant

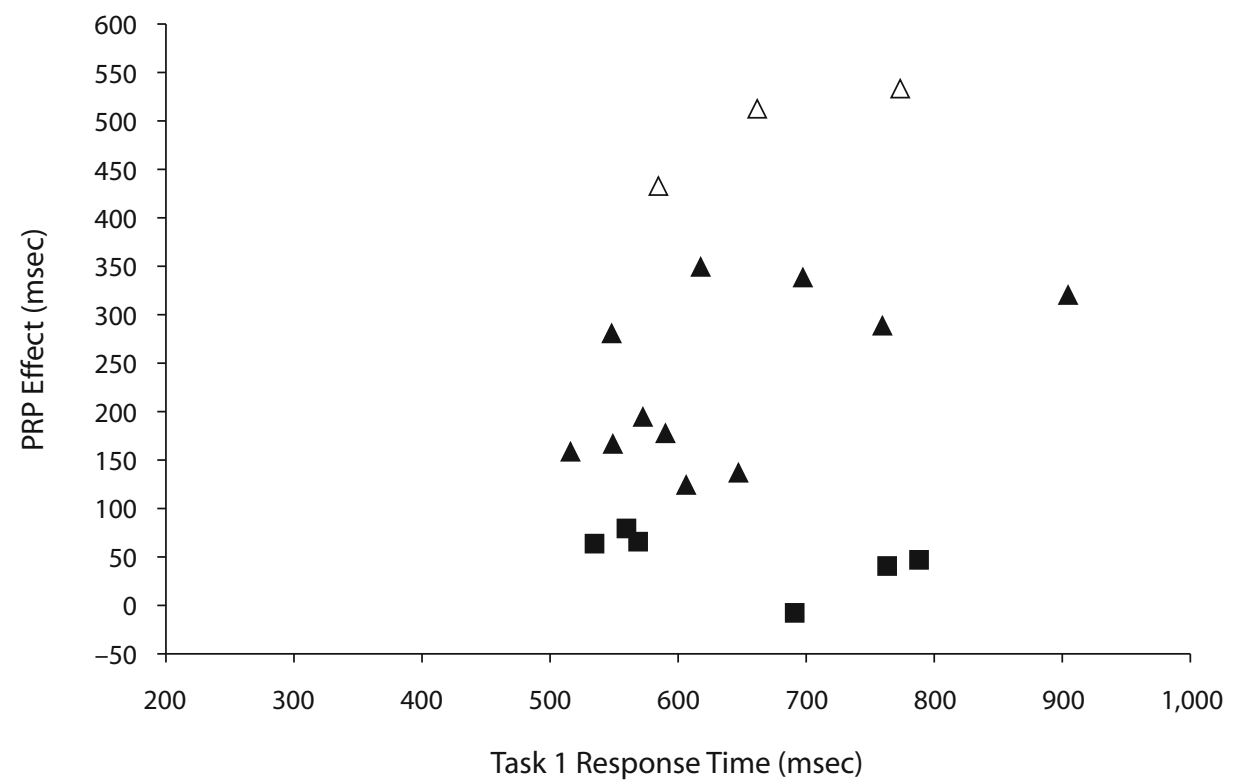

Figure 4. Psychological refractory period (PRP) effect as a function of Task 1 response time (RT1) for each participant in Experiment 1. The points representing the 6 participants suspected of bottleneck bypassing are represented by filled squares; the points representing the 3 participants who performed the Task 1 central stage followed by the Task 2 central stage (i.e., the bottleneckers) are represented by unfilled triangles; and the points representing the other 11 participants suspected of bottleneck bypassing and response grouping are represented by filled triangles. The central bottleneck model predicts a slope of roughly 1.0 for the function relating RT1 and the size of the PRP effect. $P R P^{\prime}=.324($ RT1 $)+5.717 ; r^{2}=.046$.

however, a main effect of SOA $[F(5,95)=3.74, p<.01]$, reflecting a slight tendency to make more Task 2 errors at the shortest SOA, relative to the longest SOAs; Task 2 error rates were $5.6 \%, 4.6 \%, 4.7 \%, 5.0 \%, 3.4 \%$, and $3.5 \%$ at the 15-, 65-, 150-, 250-, 550-, and 1,000-msec SOAs, respectively. There was no interaction between Task $1 \mathrm{~S}-\mathrm{R}$ compatibility and SOA $[F(5,95)=1.16, p=.37]$.

\section{Analysis of Suspected Subgroups of Participants}

The overall results of Experiment 1 were inconsistent with the central bottleneck model in which participants must finish Task 1 central stages before beginning Task 2 central stages. First, pairing a highly practiced Task 2 with an unpracticed Task 1 resulted in unusually small

\section{Carryover of Task 1 S-R Compatibility Effects to RT2}

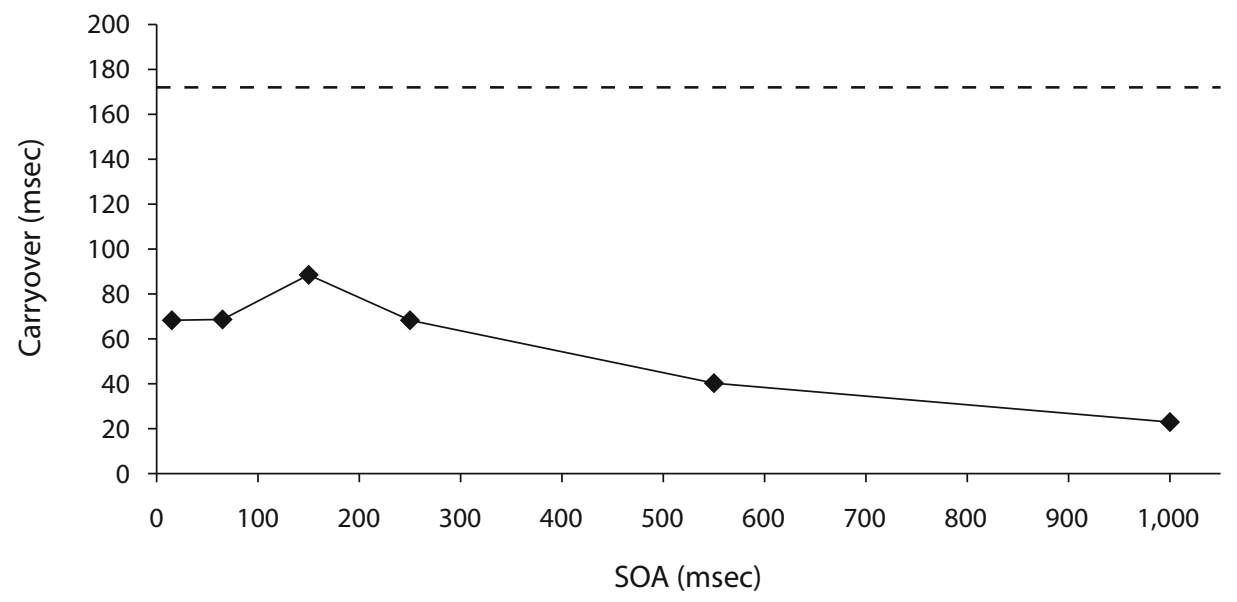

Figure 5. Effect of Task 1 stimulus-response (S-R) compatibility on Task 2 response time (RT2) at each stimulus onset asynchrony (SOA) in Experiment 1. For purposes of comparison, the mean effect of Task $1 \mathrm{~S}-\mathrm{R}$ compatibility on Task 1 response time is represented by the horizontal dashed line. 
Task 1 S-R Compatibility Effects on Response Reversal Rate

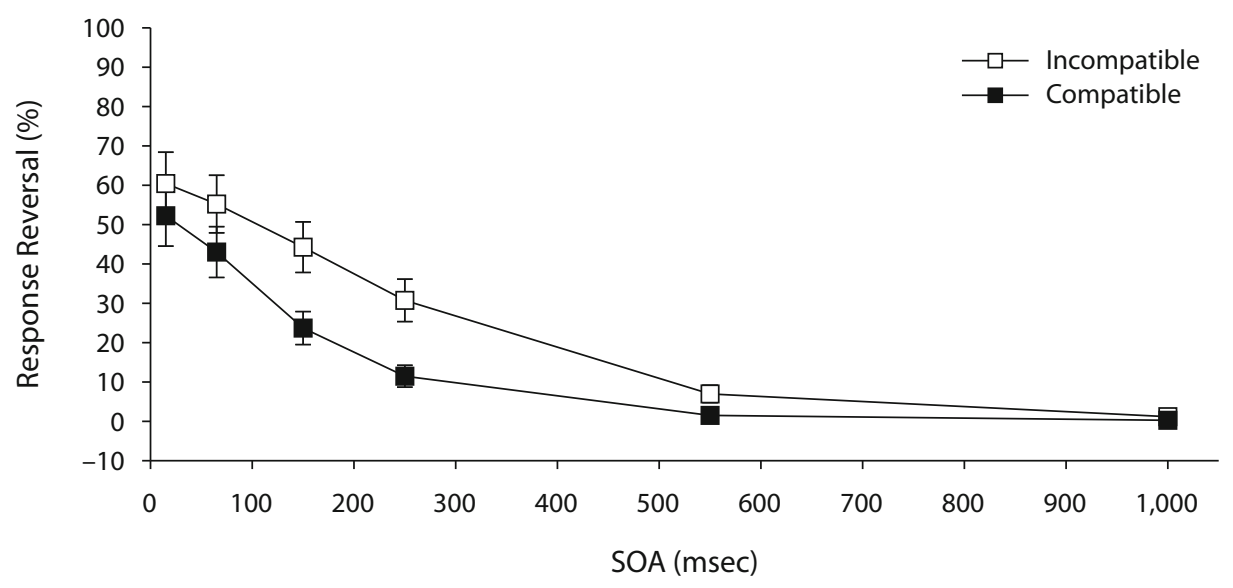

Figure 6. Effect of Task 1 stimulus-response (S-R) compatibility on the percentage of response reversal at each stimulus onset asynchrony (SOA) in Experiment 1. Bars show standard errors.

PRP effects ( $<100 \mathrm{msec}$ for 6 participants). Normally, a relatively long RT1 like that found in the present study (more than $500 \mathrm{msec}$ ) would produce a large PRP effect $(300+\mathrm{msec})$. Second, we failed to confirm the predicted positive linear relationship across participants between RT1 and the size of the PRP effect. Third, Task $1 \mathrm{~S}-\mathrm{R}$ compatibility effects (172 msec on RT1) failed to carry over fully to RT2 at the shortest SOA (the observed carryover was only $39.5 \%$ ). Fourth, the participants emitted Task 2 responses before Task 1 responses on a high percentage of trials at short SOAs (56.3\% response reversals at the 15 -msec SOA).

Although the overall data are clearly inconsistent with the standard central bottleneck model, they do not tell the whole story. For a full understanding of the data, it is necessary to consider the possibility of qualitative differences between groups of participants. Below, we will present the case that the data represent a mixture of participants who bypassed the bottleneck and participants who did not bypass the bottleneck. Whereas Ruthruff, Van Selst, et al. (2006) found evidence that a minority bypassed the bottleneck $(25 \%)$, the present study showed evidence that the majority bypassed the bottleneck (85\%).

\section{Evidence of Distinct Subgroups \\ (Bottleneckers, Suspected Bypassers, and Suspected Bypass Groupers)}

PRP effects. We began by provisionally assigning the participants to three different groups on the basis of the size of their PRP effect. We found unusually small PRP effects $(<100 \mathrm{msec})$ in 6 participants, modest PRP effects (from 124 to $349 \mathrm{msec}$ ) in 11 participants, and much larger PRP effects in 3 participants (from 433 to $533 \mathrm{msec}$ ). This breakdown based loosely on clustering of PRP effects is, admittedly, somewhat arbitrary. As will be shown below, however, there is corroborating evidence that each of these groups showed distinct patterns of results. In what follows, we will examine the key bottleneck model predictions separately for each group.
Response reversal rate. As was noted above, response reversals should be very rare if the central bottleneck is intact but should be frequent (at short SOAs) if the bottleneck has been bypassed. Table 1 shows the percentages of response reversals, mean RTS, and mean error rates on both Task 1 and Task 2 across SOAs for each of the three different groups. Consistent with the bottleneck prediction, the 3 participants with the largest PRP effects (from 433 to $533 \mathrm{msec}$ ) rarely reversed responses $(0.6 \%$ overall), further confirming that they did not bypass the

Table 1

Mean Response Reversal Rates (RRs), Mean Reaction Times to Task 1 and Task 2 (RT1s and RT2s, Respectively), and Mean Error Rates to Task 1 and Task 2 (ER1s and ER2s, Respectively) in Experiment 1 As a Function of Stimulus Onset Asynchrony (SOA) for Participants Suspected to Have Bypassed

the Bottleneck $(n=6)$, Participants Suspected to Have Bypassed the Bottleneck and Grouped Task 1 and Task 2 Responses at Short SOAs $(n=11)$, and Participants Who Did Not Bypass the Bottleneck (i.e., Bottleneckers; $\boldsymbol{n}=3$ )

\begin{tabular}{lrrrrrr}
\hline & \multicolumn{5}{c}{ Task 1-Task 2 SOA (msec) } \\
\cline { 2 - 7 } Measure & 15 & 65 & 150 & 250 & 550 & 1,000 \\
\hline Suspected bypassers & & & & & & \\
RR (in \%) & 91.1 & 81.3 & 58.0 & 36.6 & 7.5 & 1.4 \\
RT1 (in msec) & 657 & 662 & 657 & 644 & 635 & 650 \\
ER1 (in \%) & 6.9 & 5.9 & 6.3 & 6.5 & 7.0 & 5.8 \\
RT2 (in msec) & 415 & 409 & 421 & 412 & 397 & 367 \\
ER2 (in \%) & 4.5 & 3.3 & 4.6 & 4.9 & 2.6 & 2.6 \\
Suspected bypass groupers & & & & & \\
RR (in \%) & 52.4 & 44.9 & 29.8 & 18.4 & 3.6 & 0.5 \\
RT1 (in msec) & 627 & 637 & 628 & 623 & 650 & 657 \\
ER1 (in \%) & 9.8 & 7.9 & 7.8 & 6.2 & 4.6 & 4.0 \\
RT2 (in msec) & 581 & 557 & 520 & 468 & 405 & 351 \\
ER2 (in \%) & 6.1 & 5.7 & 5.3 & 5.5 & 3.8 & 4.4 \\
Bottleneckers & & & & & & \\
RR (in \%) & 1.2 & 0.3 & 1.5 & 0.0 & 0.0 & 0.3 \\
RT1 (in msec) & 658 & 648 & 649 & 665 & 692 & 725 \\
ER1 (in \%) & 3.4 & 4.2 & 3.3 & 4.2 & 2.3 & 1.9 \\
RT2 (in msec) & 953 & 901 & 818 & 763 & 577 & 460 \\
ER2 (in \%) & 6.3 & 3.4 & 2.3 & 3.2 & 3.6 & 2.3 \\
\hline
\end{tabular}


bottleneck. In sharp contrast with the bottleneck prediction, however, the other participants frequently reversed responses. At the short SOAs (15 and $65 \mathrm{msec}$ ), the response reversal rate was $86.2 \%$ for the 6 participants with the smallest PRP effects $(<100 \mathrm{msec})$ and $48.7 \%$ for the 11 participants with intermediate PRP effects (from 124 to $349 \mathrm{msec})^{2}$

To determine whether the observed response reversal rate at the 15 -msec SOA is consistent with bottleneck bypass (i.e., a parallel race between tasks), we conducted a simulation. Our approach was simply to use the observed RT1 and RT2 at the 1,000-msec SOA as a measure of how long it takes to perform each task in isolation. We then determined, for each trial, whether Task 1 would have finished after Task 2 if they raced in parallel (independently and with no interference) at the $15-\mathrm{msec}$ SOA. In other words, we checked how often RT1 exceeded RT $2+15$.

When focused on the 6 participants with the smallest PRP effects, the simulation revealed that the expected and the observed response reversal rates $(95.5 \%$ and $91.1 \%)$ were virtually identical. For the 3 participants with the largest PRP effects, however, the predicted response reversal rate $(95.2 \%)$ was much higher than the observed rate $(1.2 \%)$. Thus, the simulations provide additional evidence of bottlenecking in the 3 participants with the largest PRP effects and evidence of bottleneck bypassing in the 6 participants with the smallest PRP effects.

Interresponse intervals. Another way to distinguish between bottlenecking and bypassing is to examine the distribution of interresponse intervals (IRIs) for each participant at the shortest SOA $(15 \mathrm{msec})$. Response grouping should result in a sharp peak in IRIs near 0 msec. Bypassing (without grouping), however, should produce a broad peak of IRIs located at a value less than $0 \mathrm{msec}$ (because Task 2 was easier than Task 1). Figure 7 shows, for each subgroup, the percentage of trials at the 15-msec SOA in which the IRI fell within specific ranges. The results reveal distinct patterns for each subgroup. Clearly, the bottleneckers responded to Task 1 well before Task 2 on a very high percentage of trials, consistent with a central bottleneck. In contrast, the suspected bypassers produced a broad range of IRIs, consistent with bottleneck bypassing. The 11 participants with intermediate PRP effects, meanwhile, emitted Task 1 and Task 2 responses in close temporal succession: Almost half of the IRIs (44.6\%) fell within the narrow range from -75 to $+75 \mathrm{msec}$. Thus, the participants with intermediate IRIs appear to have been response groupers.

In principle, the participants with intermediate IRIs could have experienced a bottleneck delay and then grouped responses or bypassed the bottleneck and then grouped responses. In both cases, grouping necessarily involves withholding one of the responses for a period of time, which should elevate RTs, relative to the longest SOA (where response grouping rarely occurred, according to IRI distributions). In the case of bottleneck grouping, it should be necessary at the short SOA to withhold the Task 1 response for a substantial period of time. Thus, RT1 should be elevated at short SOAs, relative to long SOAs. In contrast to this prediction, there was no sign of RT1 lengthening at short SOAs, relative to the longest SOA, among these participants (see Table 1). We can therefore rule out bottleneck grouping in favor of bypass grouping. Note that the latter hypothesis (bypass grouping) would predict a brief withholding of the Task 2 response (which we know should usually be ready first, on the basis of our simulations). This slowing of Task 2 could explain why these participants, suspected to have bypassed the bottleneck, still showed modest PRP effects. To summarize, we classify these participants with intermediate PRP effects as suspected bypass groupers.

Task 1 carryover to RT2. As another indication of whether the bottleneck was bypassed, we computed the percentage of Task $1 \mathrm{~S}-\mathrm{R}$ compatibility effects that carried over to RT2 for the bottleneckers, the suspected

\section{Experiment 1 \\ Distribution of IRIs at the 15-msec SOA}

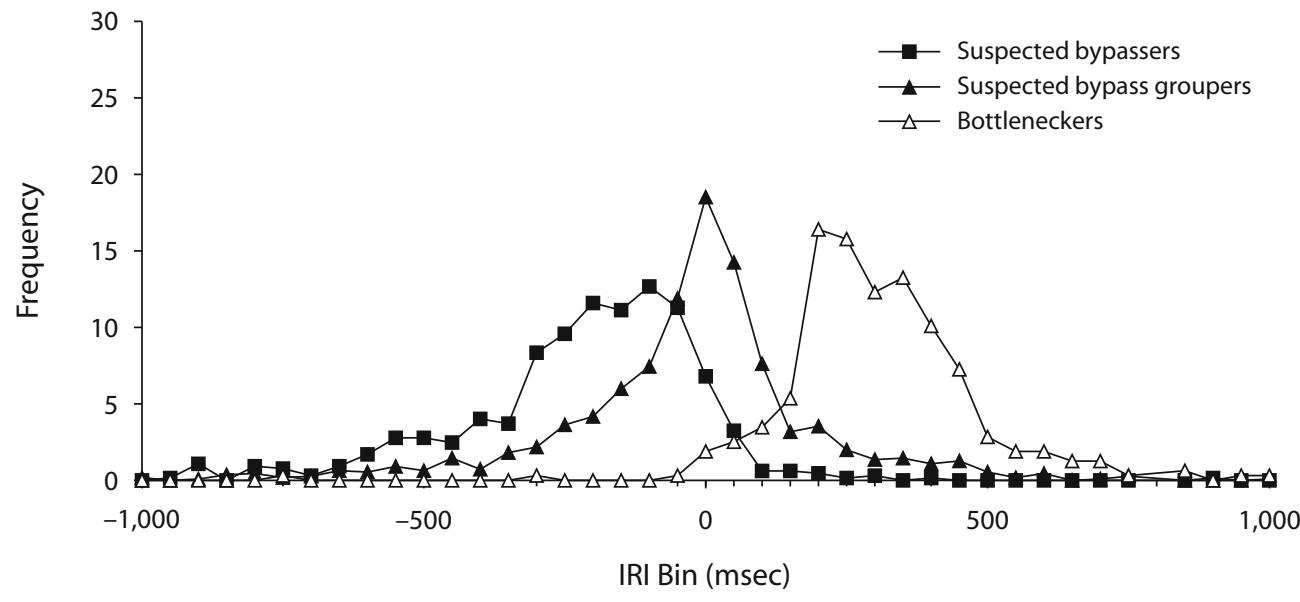

Figure 7. Histogram of interresponse intervals (IRIs) for Experiment 1 at the 15-msec stimulus onset asynchrony (SOA) for the suspected bypassers $(n=6)$, the suspected bypass groupers $(n=11)$, and the bottleneckers $(n=3)$. 
bypassers, and the suspected bypass groupers. As was described above, bottlenecking should produce full carryover. Indeed, the bottleneckers produced $87.5 \%$ carryover at the shortest SOA: The Task $1 \mathrm{~S}-\mathrm{R}$ compatibility effect was $166 \mathrm{msec}$ on RT1 and $140 \mathrm{msec}$ on RT2. For the suspected bypassers, assumed to perform Task 1 and Task 2 central operations simultaneously, there was no obvious reason for Task $1 \mathrm{~S}-\mathrm{R}$ compatibility to strongly influence RT2. As was predicted, the suspected bypassers produced only $10.1 \%$ carryover at the shortest SOA: The effect of Task 1 S-R compatibility was 199 msec on Task 1 but only $20 \mathrm{msec}$ on Task 2. An intermediate amount of carryover is predicted for the suspected bypass groupers. Carryover is expected when the Task 2 response is delayed to wait for the Task 1 response, except when the Task 2 response does not wait (either because it finished relatively late or because the participant did not group responses on that particular trial). Roughly consistent with this prediction, the suspected bypass groupers produced $47.2 \%$ carryover at the shortest SOA: The effect of Task 1 compatibility was $159 \mathrm{msec}$ on Task 1 and $75 \mathrm{msec}$ on Task 2. These findings further support our classification of participants suspected to have bypassed the central bottleneck (i.e., suspected bypassers and suspected bypass groupers) versus the participants who were unable to do so (i.e., bottleneckers). Table 2 summarizes the differential amounts of carryover between the three groups of participants, along with the other differences noted earlier.

\section{Have the Suspected Bypassers and the Suspected Bypass Groupers Simply Reversed the Central Processing Order, Leaving the Central Bottleneck Intact?}

For the 6 suspected bypassers and the 11 suspected bypass groupers, converging evidence conclusively ruled out the presence of a central bottleneck with Task 1 central stage followed by Task 2 central stage. Instead, the results were consistent with bottleneck bypassing. At a glance, however, it appears that the bottleneck model could also explain many of the key results above (e.g., small PRP effects on Task 2, frequent response reversals, little or no Task 1 carryover) simply by assuming that the participants reversed the central-processing order. This possibility seemed unlikely a priori, given instructions emphasizing Task 1 processing speed and the fact that S1 was always presented before S2. Nevertheless, it needs to be considered explicitly before concluding decisively in favor of bottleneck bypassing. In the section below, we will de- scribe and evaluate two critical predictions of a central bottleneck with a reversed central-processing order.

The first critical prediction of central bottlenecking with a reversed central-processing order is the appearance of the PRP effect on Task 1 (rather than on Task 2) for the short and intermediate SOAs, at which participants reversed response order. Inconsistent with this prediction, there was no sign of Task 1 slowing at short/intermediate SOAs, relative to the longest SOA, in the overall data (see Figure 3). As is shown in Table 1, there was also no evidence of Task 1 slowing in analyses restricted to the suspected bypassers and the suspected bypass groupers. One could argue that because Task 2 was highly practiced, it might be completed so quickly as to not interfere with Task 1 (i.e., the bottleneck might be latent). This seems unlikely given that, in the suspected bypassers and the suspected bypass groupers, baseline RT2 at the 1,000-msec SOA was not especially short (367 and $351 \mathrm{msec}$, respectively).

To more concretely estimate the size of PRP effect on Task 1 predicted by an intact central bottleneck model with a reversed central-processing order, we performed a type of simulation. The basic approach, described in detail in the Appendix, was to use the PRP:RT1 functions from related experiments in which we believe participants did face a bottleneck to estimate how much interference should have occurred in the present Experiment 1.

The first estimation was based on the present Experiment 2. This experiment will be described in detail later, but the critical things to note here are that (1) Experiment 2 reversed the task order (i.e., auditory task then visual task), and (2) the data implicate a central bottleneck in the same order hypothesized to have occurred in the present Experiment 1 . In the suspected bypassers, the estimated PRP effect on Task 1 was $58 \mathrm{msec}$ at the $15-\mathrm{msec}$ SOA, $117 \mathrm{msec}$ at the $65-\mathrm{msec}$ SOA, and $107 \mathrm{msec}$ at the $150-\mathrm{msec}$ SOA (see the Appendix for details). In the suspected bypass groupers, the estimated PRP effect on Task 1 was $43 \mathrm{msec}$ at the $15-\mathrm{msec} \mathrm{SOA}, 83 \mathrm{msec}$ at the $65-\mathrm{msec} \mathrm{SOA}$, and $72 \mathrm{msec}$ at the 150 -msec SOA. It is interesting to note that the amount of predicted Task 1 slowing was greater at the intermediate SOAs, even though the probability of response reversals was slightly lower. The reason is that if the participants did reverse the central-processing order, the amount of Task 1 slowing is greatest at intermediate SOAs because the visual task had a longer head start (i.e., had to wait longer). All of these predicted values differ significantly (.05) from the actual amount of Task 1 slowing observed in these two groups, which was negligible.

Table 2

Differential Results Between the Three Subgroups of Participants in Experiment 1

\begin{tabular}{lccc}
\hline \multicolumn{1}{c}{ Measure } & $\begin{array}{c}\text { Bottleneckers } \\
(n=3)\end{array}$ & $\begin{array}{c}\text { Suspected } \\
\text { Bypass Groupers } \\
(n=11)\end{array}$ & $\begin{array}{c}\text { Suspected } \\
\text { Bypassers } \\
(n=6)\end{array}$ \\
\hline PRP effect & $493 \mathrm{msec}$ & $230 \mathrm{msec}$ & $48 \mathrm{msec}$ \\
Response reversals at the 15-msec SOA & $1.2 \%$ & $52.4 \%$ & $91.1 \%$ \\
Carryover of Task 1 compatibility effects to RT2 & $87.5 \%$ & $47.2 \%$ & $10.1 \%$ \\
\hline Note-PRP, psychological refractory period; SOA, stimulus onset asynchrony; RT2, Task 2 reaction \\
time.
\end{tabular}


Similar conclusions follow if we, instead, use the equation relating the PRP effect and RT1 across sessions in Van Selst et al. (1999). They adopted tasks very similar to those used in Experiment 1, but in the opposite order (the order used in our Experiment 2), and concluded in favor of a processing bottleneck. Their shortest SOA was also nearly identical to ours (17 vs. $15 \mathrm{msec}$ ). Using their equation, the estimated PRP effect on Task 1 at the 15 -msec SOA was $115 \mathrm{msec}$ for the suspected bypassers and $72 \mathrm{msec}$ for the suspected bypass groupers. Again, these values are significantly greater than the negligible amount of slowing observed in these two groups. These calculations argue against a central bottleneck with the reversed central-processing order and, instead, support our conclusion in favor of bottleneck bypassing in these 17 participants.

The second critical prediction derived from the intact central bottleneck model, with frequent reversals of the central-processing order, predicts a bimodal IRI distribution: one mode with large, negative IRIs in which the Task 2 central stage is followed by the Task 1 central stage, and another mode with large, positive IRIs in which the Task 1 central stage is followed by the Task 2 central stage. Grouping would add a third mode with IRIs near $0 \mathrm{msec}$, resulting in a trimodal distribution. Indeed, this is exactly what has been observed early in practice in designs that mixed positive and negative SOAs (see Pashler, 1994b; Ruthruff, Pashler, \& Hazeltine, 2003). To test this prediction, we focused on SOAs for which the two response orders were roughly equally likely to occur (see Figure 8, upper panel for the suspected bypassers and lower panel for the suspected bypass groupers): The 150 -msec SOA (58.0\% response reversals) and the 250 msec SOA (36.6\% response reversals) for the suspected bypassers; the 15 -msec SOA (52.4\% response reversals) and the $65-\mathrm{msec} \mathrm{SOA}$ (44.9\% response reversals) for the suspected bypass groupers. Thus, the IRI distributions for these SOAs should show evidence of at least two modes (one for each central-processing order). In contrast with this prediction, Figure 8 clearly indicates a broad, unimodal IRI distribution at the 150- and 250-msec SOAs for the suspected bypassers. This is exactly what one would expect if the processes for the two tasks bypassed the central bottleneck and, thus, were performed in parallel. The suspected bypass groupers, at the 15- and 65-msec SOAs, showed a sharp peak near $0 \mathrm{msec}$, consistent with grouping. Neither of these two groups exhibited a trend toward a mode with large, positive IRIs, as demonstrated by the three bottleneckers (see Figure 7), or a trend toward a mode with large, negative IRIs.

\section{Discussion}

In Experiment 1, pairing a highly practiced auditoryvocal Task 2 with an unpracticed visual-manual Task 1 provided overall results inconsistent with the intact central bottleneck model in which the Task 1 central stage was followed by Task 2 central stages. Because the data suggested categorical differences among the participants, subsequent analyses were performed separately for the participants with large PRP effects (from 433 to $533 \mathrm{msec}$; $n=3$ ), intermediate PRP effects (from 124 to $349 \mathrm{msec}$; $n=11)$, and small PRP effects ( $<100 \mathrm{msec} ; n=6)$. Detailed analyses indicated that the participants with large PRP effects were subject to the standard processing bottleneck encountered in numerous previous PRP studies (with central operations on Task 1 performed before those on Task 2). There was no evidence of such a bottleneck, however, for the participants with small and intermediate PRP effects (which differed mainly in the use of a response grouping strategy at short SOAs).

We then considered the possibility that the central bottleneck was intact, even for the participants with small and intermediate PRP effects on RT2, but that the centralprocessing order was simply reversed. This possibility was inconsistent with two additional lines of evidence in Experiment 1 (the lack of PRP effects on Task 1 and the lack of a bimodal or trimodal distribution of IRIs). Thus, the data indicate that these two groups, who represented the vast majority (17 out of 20 ) of the participants, genuinely bypassed the central-processing bottleneck.

\section{EXPERIMENT 2 Transfer to a PRP Design With the Reverse Task Order}

Ruthruff, Van Selst, et al. (2006) found evidence of bottleneck bypassing in a small proportion of participants (4 of 18) when the tone task was used as Task 2 (as in the present Experiment 1), but not when it was used as Task 1 (0 of 18). To explain this observation, they proposed the greedy resource recruitment hypothesis. This hypothesis asserts that automatized tasks, which do not need access to central resources, still recruit those resources when available. In other words, an automatized task might still engage central resources when it serves as Task 1 and, thus, force a nonautomatized Task 2 to experience a bottleneck delay.

Although this hypothesis fit their data, they acknowledged that it was post hoc. Direct evidence would require showing that individual participants bypassed the bottleneck with one task order, but not with the other. However, none of the bypassers in Ruthruff, Van Selst, et al. (2006) were ever tested with the opposite task order. Accordingly, it is conceivable that the differential proportion of bypassers simply reflected luck in selecting participants. Also, when the easier task (tone task) served as Task 1 (Experiment 1), it was difficult for them to rule out the possibility of a latent bottleneck (Lien et al., 2006; Ruthruff, Johnston, et al., 2003), in which Task 1 central operations were completed so quickly that they rarely had a chance to conflict with Task 2 central operations. So, it is possible that they underestimated the number of bypassers in Experiment 1.

As a direct test of the greedy resource recruitment hypothesis, we asked the participants from Experiment 1 with the strongest evidence of having bypassed the bottleneck (i.e., the 6 bypassers) to complete a follow-up experiment in which we reversed the roles of the two tasks in the dualtask sessions. The auditory task became Task 1, and the visual task became Task 2 . Because these participants had 


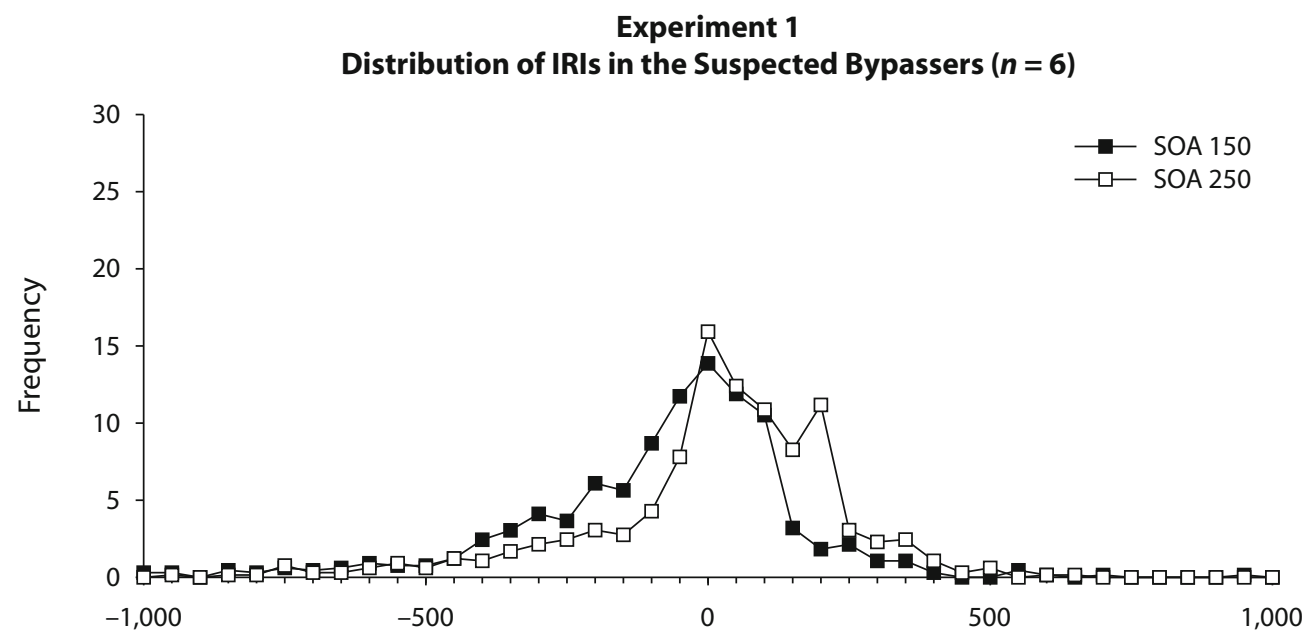

IRI Bin (msec)

Experiment 1

Distribution of IRIs in the Suspected Bypass Groupers $(n=11)$

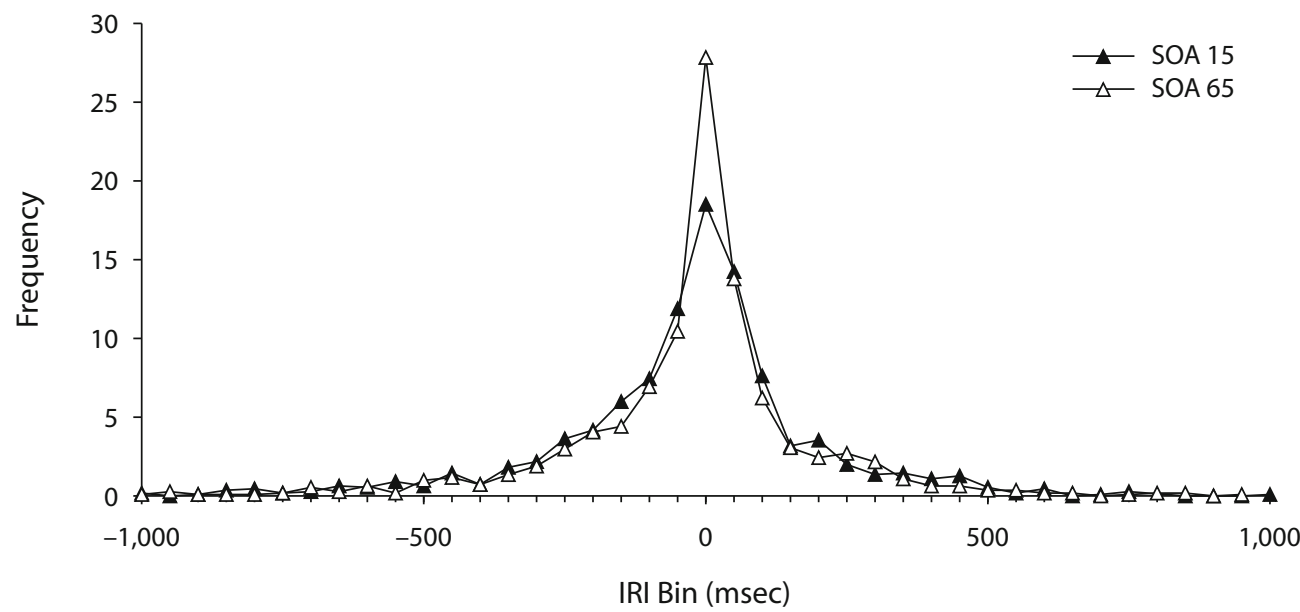

Figure 8. Histogram of interresponse intervals (IRIs) for Experiment 1 at stimulus onset asynchronies (SOAs) for which Task 1 and Task 2 response orders were used roughly equally often-that is, at the 150and 250-msec SOAs for the suspected bypassers $(n=6$, upper panel) and at the 15- and 65-msec SOAs for the suspected bypass groupers $(n=11$, lower panel).

already practiced Auditory Task 1, no further single-task practice was given prior to the dual-task sessions.

To deal with the latent bottleneck issue, we simply gave Task 2 a seemingly generous head start of 150 msec. In other words, we subtracted $150 \mathrm{msec}$ from the set of SOAs used in Experiment 1. This shift should increase the overlap in the demand for the central operations of Task 1 and Task 2 - in particular, at the shortest SOA of $-135 \mathrm{msec}$. It would have been possible to use an even larger offset (say, $300 \mathrm{msec}$ ), commensurate with the difference in mean RT for the two tasks. However, we were concerned that with such a large offset, the participants would notice the reversal of the presentation order and would begin deliberately selecting responses in the opposite order.

According to the greedy resource recruitment hypothesis, Task 1 should recruit central resources (even though it does not need them), thus making those resources unavailable to Task 2. Consequently, Experiment 2 should produce PRP effects proportionate to mean RT1 and other signs of a central bottleneck.

\section{Method}

The participants $(M=25$ years, $S D=1.7$ years, range $=24-28$ years; 1 woman) were those who had clearly bypassed the bottleneck in Experiment 1: G.H., R.D., T.D., M.D., and Y.P. (1 of the 6 original bypassers from Experiment 1 was unavailable to complete Experiment 2). They performed three dual-task transfer sessions (384 experimental trials per session, for a grand total of 1,152 trials). They were paid for their participation (\$10 Canadian per session).

The method was identical to that in Experiment 1, except for the three following aspects. First, no single-task practice sessions were given (the Auditory Task 1 was already highly practiced); the participants simply performed three dual-task transfer sessions on 
3 nonconsecutive days of the same week. Second, we reversed the roles of the tone task (now Task 1) and the visual task (now Task 2) in the dual-task condition. Accordingly, the instructions emphasized responding as quickly and accurately as possible to each task while emphasizing the speed of Auditory Task 1. Third, $150 \mathrm{msec}$ were subtracted from each of the six SOAs in Experiment 1, leaving intact the SOA range but producing a new SOA set: $-135,-85,0,100$, 400 , and $850 \mathrm{msec}$. Although two of the SOAs were now negative SOAs, Task 1 still came first or simultaneously with Task 2 on $67 \%$ of the trials, and Task 1 was still emphasized in the instructions.

\section{Analyses}

We conducted separate ANOVAs on mean RT1, RT2, response reversal rate, Task 1 error rate, and Task 2 error rate in the dual-task transfer sessions (Sessions 11 and 12), using the factors of SOA and Task 2 S-R compatibility (compatible vs. incompatible) as withinsubjects variables. Only dual-task trials with correct responses and latencies between 100 and 2,500 msec on both Task 1 and Task 2 were included in the RT analysis. Application of the RT cutoffs led to the removal of $2.58 \%$ of the dual-task trials from the analysis.

\section{Results}

\section{Tone Task 1 RTs in the Transfer Sessions}

Figure 9 shows mean RT1 and RT2 as a function of SOA, averaged across the last two transfer sessions (i.e.,
Sessions 11 and 12). Individual mean RT1s for participants G.H., R.D., T.D., M.D., and Y.P. were 276, 346, 320, 401, and $263 \mathrm{msec}$, respectively. Mean RT1 was unaffected by SOA $[F(5,20)=1.29, p=.31]$. This finding supports our contention that the participants gave Task 1 priority, despite the fact that the Task 2 stimulus actually came first on $33 \%$ of the trials. Also, mean RT1 was not affected by Task 2 S-R compatibility $[F(1,4)<1]$. This finding, combined with the lack of an SOA effect, indicates that the participants rarely grouped responses together (note that these participants also did not often group responses in Experiment 1). Finally, the two-way interaction between SOA and Task 2 S-R compatibility was not significant $[F(5,20)<1]$.

\section{PRP Effect on Visual Task 2 in the Transfer Sessions}

The PRP effect was computed as the difference between RT2 at the $-135-\mathrm{msec}$ SOA $(M=666 \mathrm{msec}, S D=$ $118 \mathrm{msec})$ and the $850-\mathrm{msec} \mathrm{SOA}(M=578 \mathrm{msec}, S D=$ $89 \mathrm{msec})$. Other things being equal, one would expect much smaller PRP effects in this experiment than in Ex-

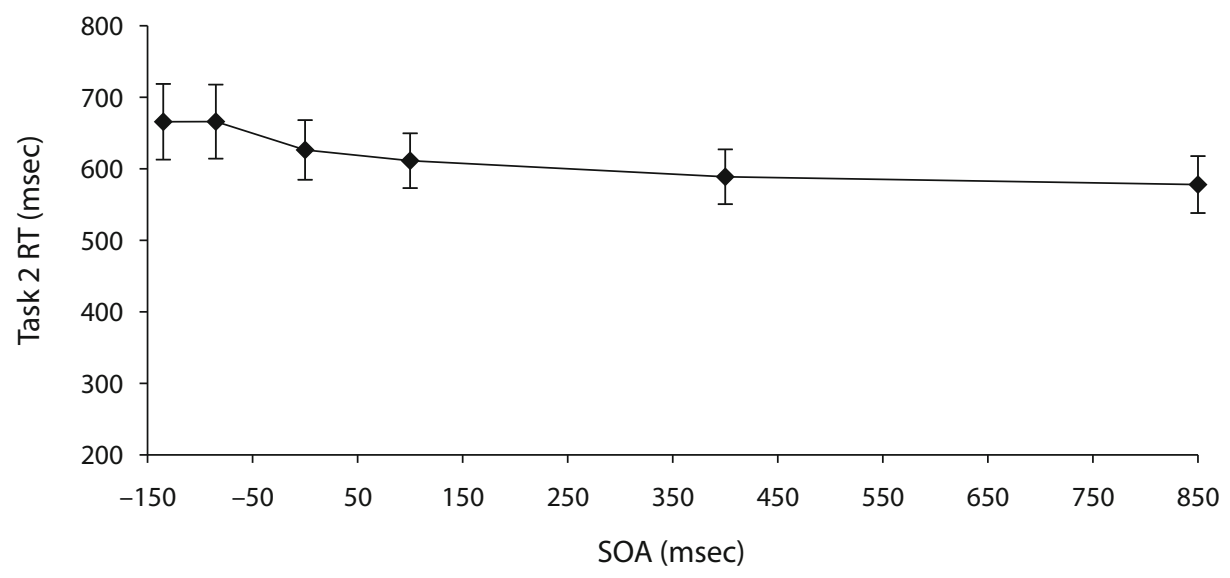

RT1

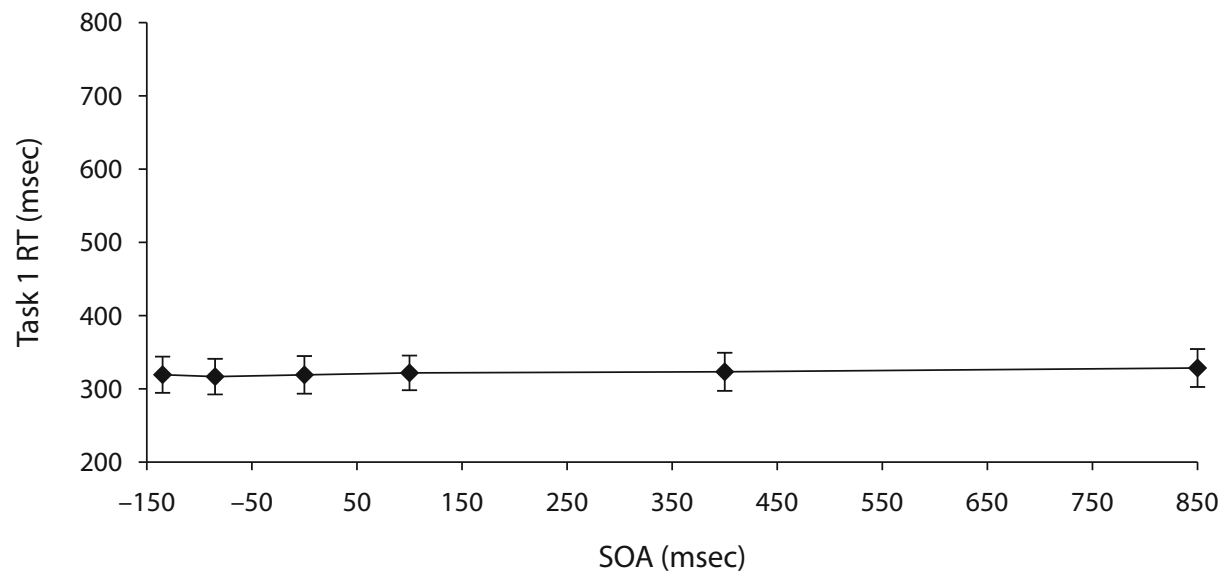

Figure 9. Mean Task 2 and Task 1 response times (RTs) in Experiment 2 as a function of stimulus onset asynchrony (SOA). Bars show standard errors. 


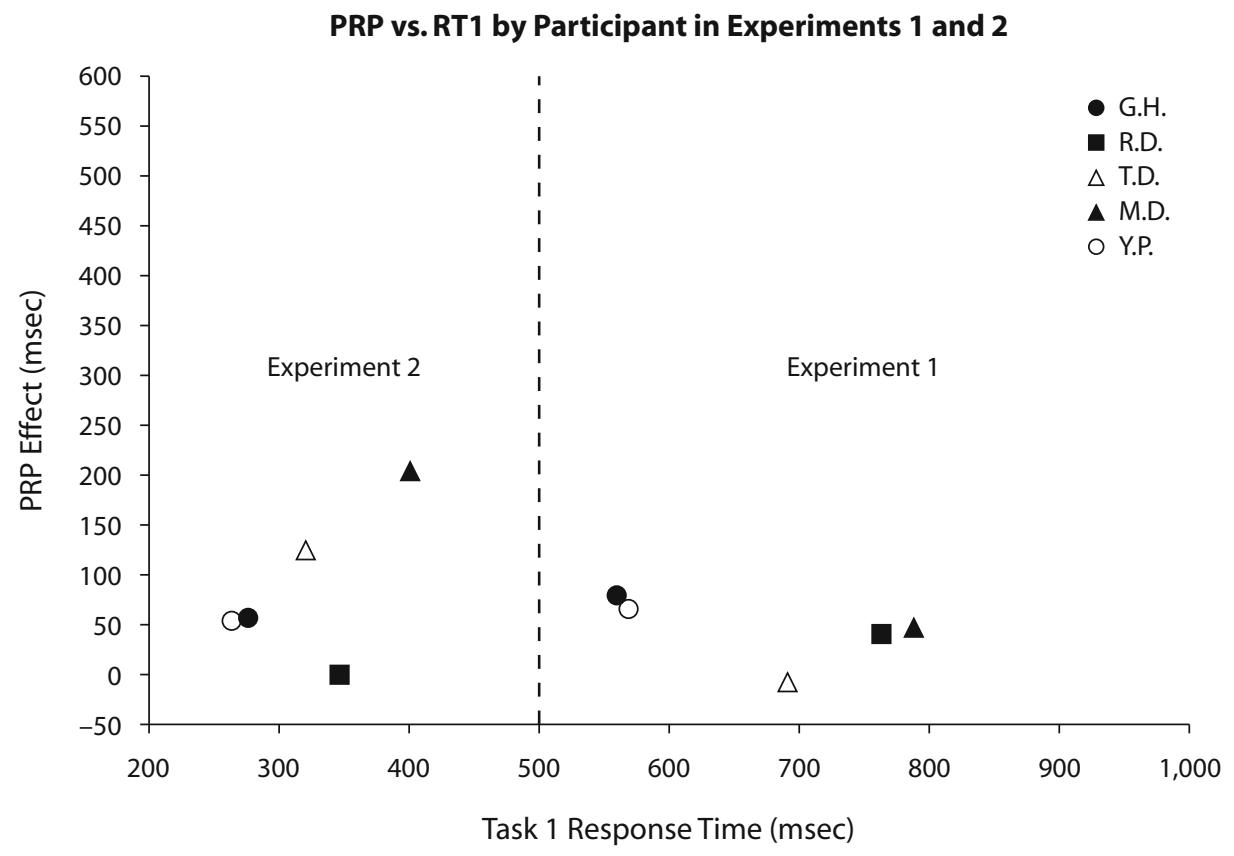

Figure 10. Psychological refractory period (PRP) effect as a function of Task 1 response time (RT1) for each of the 5 participants in Experiment 2 (left of the vertical dashed line) and Experiment 1 (right of the vertical dashed line). The central bottleneck model predicts a slope of 1.0 for the function relating RT1 and the size of the PRP effect. In Experiment 1, PRP' $=-.158($ RT1) + 151.560; $r^{2}=.257$. In Experiment 2, $\mathrm{PRP}^{\prime}=.839(\mathrm{RT} 1)-181.814 ; r^{2}=.351$.

periment 1 because of the much shorter mean RT1 (321 vs. $674 \mathrm{msec}$ ). Note that this difference in mean RT1s (353 msec) far exceeds the 150-msec compensatory shift in the SOAs. However, the size of the PRP effect was numerically greater in Transfer Sessions 11 and 12 of this experiment $(M=88 \mathrm{msec}, S D=79 \mathrm{msec})$ than it was for these same participants in Test Sessions 8 and 9 of Experiment $1(M=45 \mathrm{msec}, S D=33 \mathrm{msec})$. This difference between experiments was not significant $[t(4)=$ $1.02, p=.37]$.

\section{PRP Effect Versus RT1 for Individuals}

Figure 10 shows the PRP effect (Experiment 2) as a function of RT1 for each participant during Transfer Sessions 11 and 12 (as well as during Test Sessions 8 and 9 of Experiment 1, for purposes of comparison).

According to the bottle neck model, combined with the assumption that individuals differ mainly in the duration of central stages (Van Selst et al., 1999), the PRP effect should be linearly related to RT1 with a slope of about 1.0. Roughly consistent with this prediction, the slope of the PRP:RT1 function was $.839\left(r^{2}=.351\right)$, a value that did not differ significantly from $1.0[t(3)<1]$. Note that the same 5 participants actually produced a negative slope in Experiment $1\left(-.158 ; r^{2}=.257\right)$.

\section{Task 2 Additivity Prediction}

The data confirmed the intact bottleneck prediction that Task 2 S-R compatibility effects should combine additively with SOA. Mean RT2 was longer in the incompatible condition $(M=699 \mathrm{msec})$, relative to the compatible condition $(M=552 \mathrm{msec})[F(1,4)=20.56, p<.05]$. The effect of Task 2 S-R compatibility was 163, 167, 146, 148, 134 , and $126 \mathrm{msec}$ at the $-135-,-85-, 0-, 100-, 400-$, and $850-\mathrm{msec}$ SOAs, respectively. The small trend toward overadditivity was significant neither overall $[F(5,20)=$ $2.18, p=.10]$ nor when just the shortest and the longest SOAs were compared $[F(1,4)=5.53, p=.08]$. This approximate additivity, however, does not necessarily indicate that the central bottleneck was present. One would also expect to observe an additive relationship if the participants had bypassed the bottleneck.

\section{Dependencies Between RT2 and RT1 Divided Into Quintiles}

Another indication of the presence of a central bottleneck comes from the dependencies between RT1 and RT2 at the shortest SOAs (see Pashler, 1994b): Random variation in the duration of Task 1 precentral and central stages should carry over to RT2. This prediction is a corollary of the Task 1 carryover prediction, applied to random variation in RT1, rather than to the systematic variation induced by a manipulation (Experiment 2 did not include a Task 1 difficulty manipulation). In contrast, the bottleneck bypass hypothesis provides no reason to expect variation in Task 1 precentral and central stages to carry over strongly to RT2 at the shortest SOA. A small amount of carryover, however, could occur due to variation in general arousal that affects both tasks the same way.

To assess the strength of the relationship between RT1 and RT2, we sorted RT1 into five bins for each participant at the -135 - and $850-$ msec SOAs. We then computed the 
mean RT2 for each RT1 bin at these SOAs. For purposes of comparison, we repeated this procedure for the 15- and 1,000-msec SOAs in Experiment 1, using the same 5 participants. Figure 11 shows the results of this analysis, averaged across the 5 participants, for Experiment 1 (left panel) and Experiment 2 (right panel). At the -135 -msec SOA, RT2 increased by $36 \mathrm{msec}$, from $611 \mathrm{msec}$ at the first RT1 quintile $(245 \mathrm{msec})$ to $647 \mathrm{msec}$ at the fifth RT1 quintile $(372 \mathrm{msec})$. At the 850 -msec SOA, RT2 increased by only $14 \mathrm{msec}$, from $543 \mathrm{msec}$ at the first RT1 quintile $(261 \mathrm{msec})$ to $557 \mathrm{msec}$ at the fifth RT1 quintile (387 msec). The slopes of the functions relating RT2 and RT1 quintile were numerically larger at the shortest SOA (.300) than at the long SOA (.213). Although the difference was not significant with only 5 participants $[t(4)<1]$, the trend is loosely consistent with a central bottleneck that occurs on at least a proportion of trials. Note that it is unrealistic to expect a perfect slope of 1, because (1) the bottleneck could be latent for especially short RT1s and (2) some of the variation in RT1 could be due to variation in the duration of response stages (Stage 1C in Figure 1), which would not carry over to RT2.

In Experiment 1, however, there was virtually no RT2 increase $(2 \mathrm{msec})$ across RT1 quintiles at the shortest SOA: RT2 was $402 \mathrm{msec}$ at the first RT1 quintile $(454 \mathrm{msec})$ and $404 \mathrm{msec}$ at the fifth RT1 quintile $(845 \mathrm{msec})$. At the 1,000-msec SOA, RT2 increased by $44 \mathrm{msec}$, from $352 \mathrm{msec}$ at the first RT1 quintile (452 msec) to $396 \mathrm{msec}$ at the fifth RT1 quintile $(889 \mathrm{msec})$. Also, the slope relating RT2 and RT1 quintiles was smaller at the $15-\mathrm{msec}$ SOA (.008) than at the 1,000-msec SOA (.115), although the difference was only marginally significant $[t(4)=$ $2.19, p=.09]$. Therefore, the results from Experiment 1 are inconsistent with a central bottleneck.
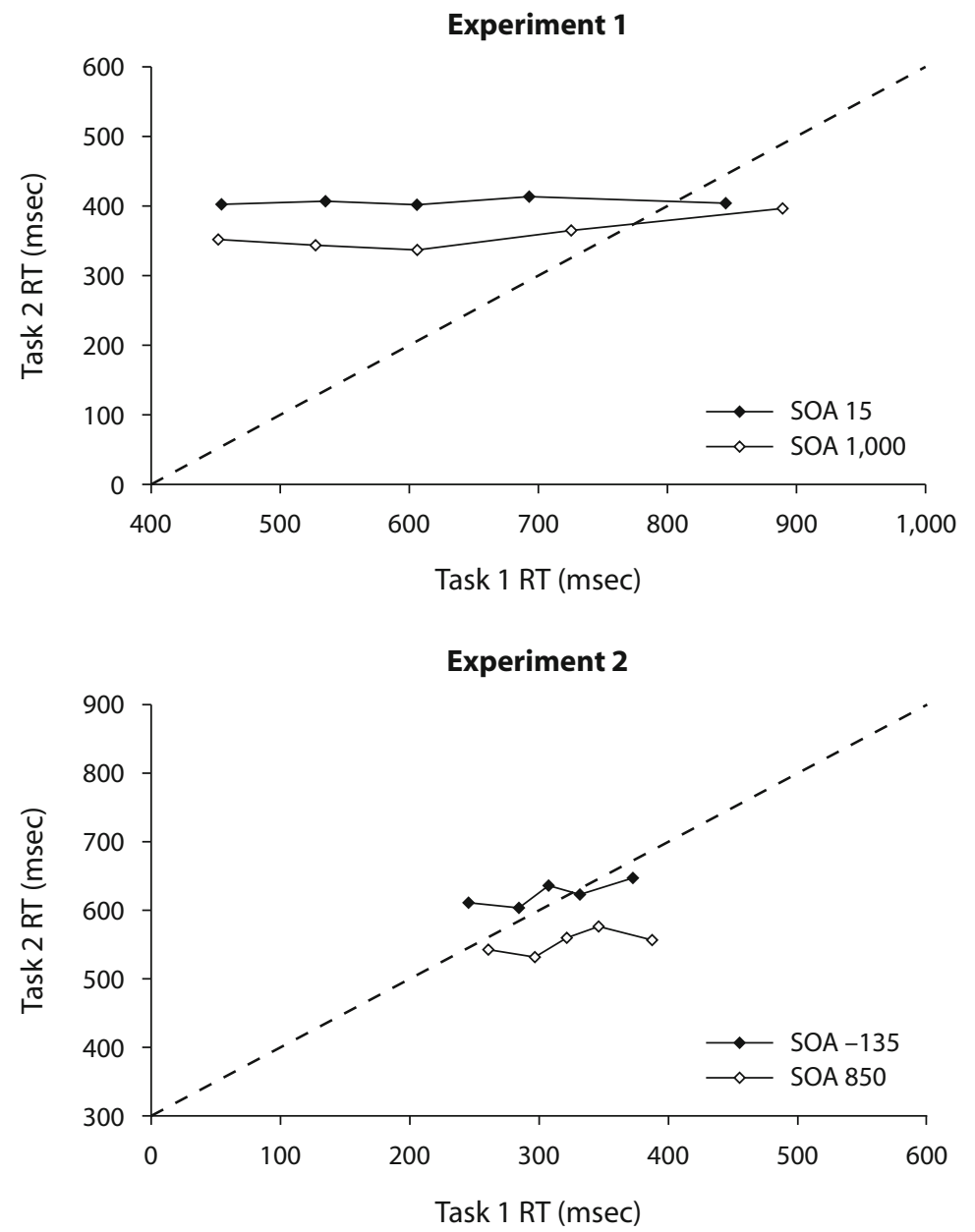

Figure 11. Task 2 response time (RT) as a function of Task 1 RT at the 15- and 1,000-msec stimulus onset asynchronies (SOAs) in Experiment 1 (top panel) and at the -135- and 850-msec SOAs in Experiment 2 (bottom panel). For each SOA, the five data points correspond to the five RT1 quintiles. In each panel, the dashed line indicates a slope of 1. Experiment 1: At the 15-msec SOA, $\mathrm{RT}^{\prime}=.008(\mathrm{RT} 1)+400.96, r^{2}=.058$; at the $1,000-$ msec SOA, RT2' $=.115$ $($ RT1 $)+285.08, r^{2}=.710$. Experiment 2: At the $-135-$ msec SOA, RT2 ${ }^{\prime}=.300$ (RT1) + 531.70, $r^{2}=.651$; at the 850-msec SOA, RT2' = .213 (RT1) + 484.80, $r^{2}=.358$. 


\section{Response Order}

The participants rarely reversed responses (Task 2 response before Task 1 response) in this experiment (0.6\%), despite the use of negative SOAs ( -135 and $-85 \mathrm{msec})$, which gave Task 2 a substantial head start. The percentage of response reversal for participants G.H., R.D., T.D., M.D., and Y.P. was $0.6 \%, 0.4 \%, 1.2 \%, 0.7 \%$, and $0.2 \%$, overall, and $0.0 \%, 0.0 \%, 1.8 \%, 0.9 \%$, and $0.0 \%$ of the trials at the -135 -msec SOA, respectively. These data provide no evidence of bottleneck bypassing.

One could argue that these data, by themselves, do not provide evidence against bottleneck bypassing. Because mean RT2 at the longest SOA $(M=578 \mathrm{msec}$, $S D=89 \mathrm{msec}$, range $=472-696 \mathrm{msec})$ was much longer than mean RT1 on average $(M=321 \mathrm{msec}, S D=$ $51.2 \mathrm{msec}$, range $=263-401 \mathrm{msec}$ ), one could question whether Task 2 had much of a chance to win a parallel race, even with a $135-\mathrm{msec}$ head start. To shed light on this issue, we used the same procedure as that employed in Experiment 1. To reiterate, our approach was to use the observed RTs at the longest SOA (where the tasks should be performed essentially independently), to estimate what would happen if they raced in parallel (with no interference) at the -135-msec SOA. Recall that, in Experiment 1 , this simulation technique correctly predicted the observed rate of response reversals, consistent with bottleneck bypassing.

In contrast with the observed $0.5 \%$ response reversal rate at the $-135-\mathrm{msec} \mathrm{SOA}$, the simulation indicated that, overall, bottleneck bypassers should have reversed responses on $16.7 \%$ of the trials at the -135 -msec SOA. Even though there were only 5 participants, this difference was marginally significant $[t(4)=2.19, p=.09]$. The expected percentage of response reversals at the -135-msec SOA for participants G.H., R.D., T.D., M.D., and Y.P. was $15.9 \%, 2.5 \%, 7.3 \%, 42.8 \%$, and $14.9 \%$ of the trials, respectively. Thus, the predicted rate was higher than the observed rate for every participant (significant by a sign test). The simulated results suggest that the virtual absence of observed response reversals in Experiment 2 is more consistent with bottlenecking than with bypassing.

\section{Task 1 and Task 2 Error Rates}

Auditory Task 1. There were no significant effects in the analysis of Task 1 error rates. Task 1 error rates were $2.5 \%$ and $3.5 \%$ in the compatible and incompatible Task 2 $\mathrm{S}-\mathrm{R}$ conditions, respectively. Also, Task 1 error rates were $4.1 \%, 2.7 \%, 2.4 \%, 2.6 \%, 2.7 \%$, and $3.5 \%$ at the $-135-$, -85-, 0-, 100-, 400-, and 850-msec SOAs, respectively.

Visual Task 2. The participants made more errors on Task 2 in the incompatible S-R condition $(10.8 \%)$ than in the compatible S-R condition $(5.2 \%)[F(1,4)=$ $30.71, p<.01]$. There was neither a main effect of SOA $[F(5,20)<1]$ nor a two-way interaction between SOA and Task $2 \mathrm{~S}-\mathrm{R}$ compatibility $[F(5,20)=1.75, p=$ $.168]$. Task 2 error rates were $7.7 \%, 6.2 \%, 9.0 \%, 9.4 \%$, $8.7 \%$, and $7.1 \%$ at the $-135-,-85-, 0-, 100-, 400-$, and 850 -msec SOAs, respectively.

\section{Discussion}

In Experiment 2, 5 participants identified as bypassers in Experiment 1 were transferred to dual-task sessions with the exact same tasks as before, but in a different order (Auditory Task 1 and Visual Task 2). The result was a mean PRP effect of $88 \mathrm{msec}$ (range $=0-204 \mathrm{msec}$ ). Although small, this PRP effect is about as large as would be predicted by the central bottleneck model, given the short mean RT1 $(M=321 \mathrm{msec})$. In fact, this 88 -msec PRP effect was numerically greater than the 45-msec PRP effect exhibited by the same participants in Experiment 1, even though mean RT1 was 353 msec shorter. This result may be viewed as a first indicator, albeit inconclusive by itself, of the presence of a bottleneck preventing Task 1 and Task 2 central operations from being carried out simultaneously.

We also found some evidence of the positive relationship between mean RT1 and the PRP effect predicted by the central bottleneck model: The slope relating the PRP effect and RT1 across participants was $.839\left(r^{2}=.351\right.$; see Figure 10). In Experiment 1, in contrast, we found no such relationship among these 5 participants: The slope was actually negative $\left(-.158 ; r^{2}=.257\right)$. It should be noted, however, that the estimated slope was not robust with a modest number of participants (e.g., the slope would decrease sharply if participant M.D. were removed and would increase if participant R.D. were removed). Thus, this PRP/ RT1 relationship is inconclusive when considered in isolation but lends some support to the bottleneck model.

Another indication of the presence of a central bottleneck was that, at the shortest SOA, RT2 was numerically greater for bins in which RT1 was greater (more so than at the longest SOA). Such a result is roughly consistent with the specific prediction of the central bottleneck model that variation in Stages 1A and 1B should carry over to RT2 at the shortest SOA. This conclusion is strengthened by the absence of a dependency between RT2 and RT1 quintiles shown by the same participants in Experiment 1.

The last indication of the presence of a central bottleneck stems from a comparison of the observed and the estimated response reversal rates. We estimated via simulation that, if the participants bypassed the bottleneck, they would reverse responses on $16.7 \%$ of the trials at the -135 -msec SOA. In contrast to this prediction, the participants actually reversed responses on only $0.5 \%$ of the trials. Note that exactly the same simulation procedure correctly predicted the observed response reversal rate in Experiment 1. Thus, the virtual absence of response reversals in Experiment 2 supports the central bottleneck model and argues against bottleneck bypassing.

Although each of these lines of evidence, by itself, is inconclusive, they sum to provide a reasonably strong case that the 5 participants who were able to bypass the central bottleneck in Experiment 1 were unable to do so reliably in Experiment 2. Experiment 2 thus provides the first a priori support for the greedy resource recruitment hypothesis, proposed a posteriori by Ruthruff, Van Selst, et al. (2006). Additional research is needed to more thoroughly test this hypothesis (e.g., by determining whether the finding generalizes to other tasks). 


\section{GENERAL DISCUSSION}

The present study was designed to determine whether bottleneck bypassing is a genuine phenomenon. Many previous practice studies have produced inconclusive results either because it was difficult to demonstrate that the bottleneck was absent, rather than merely latent (Hazeltine et al., 2002; Schumacher et al., 2001), or because bypassing was observed in only a minority of participants (Ruthruff, Van Selst, et al., 2006). The experiments were also designed to determine whether bottleneck bypassing occurs only when the easier task (subject to automatization) is presented second. According to the greedy resource recruitment hypothesis proposed a posteriori by Ruthruff, Van Selst, et al., an automatized task presented second will proceed without delay, even if a nonautomatized task presented first appropriates central resources (as in the present Experiment 1). But the same automatized task presented first (as in the present Experiment 2) will "greedily" appropriate available central resources (even though it does not need them), preventing a subsequent nonautomatized task from gaining access to these resources and, thus, producing a bottleneck delay.

Experiment 1 consisted of a single-task training phase followed by a dual-task test phase. During the training phase, the participants performed six training sessions (5,040 training trials) on a tone classification task (which would serve as Task 2 in the subsequent test sessions). During the test phase, the participants performed three dual-task sessions, pairing an unpracticed Visual Task 1 with the highly practiced Auditory Task 2 .

\section{Can a Highly Practiced Task 2 Bypass the Central Bottleneck?}

Experiment 1 provided strong support for the hypothesis that, under special circumstances, the central bottleneck can be bypassed. Several converging indicators showed that the majority of the participants $(85 \%)$ were able to bypass the central bottleneck. In particular, these participants exhibited unusually small PRP effects, produced incomplete carryover of Task $1 \mathrm{~S}-\mathrm{R}$ compatibility effects to RT2, and reversed the response order (Task 2 before Task 1) on a high percentage of the trials. These results convincingly rule out the standard bottleneck model, in which Task 1 central operations must be carried out before Task 2 central operations. Two other results (the lack of a PRP effect on Task 1 and unimodal IRI distributions) argued against an alternative bottleneck model in which the central-processing orders are often reversed. Among these bottleneck bypassers (17 participants), categorical differences also appeared: Six of them (called bypassers) consistently responded to Task 2 well before Task 1 at the shortest SOAs (without grouping responses), whereas the other 11 participants (called bypass groupers) consistently emitted responses as a couplet.

The evidence for bottleneck bypassing is especially impressive in this study (as compared with previous studies) because (1) one of the tasks (i.e., Visual Task 1) still produced very long RTs (arguing against a latent bottleneck), (2) the lack of interference was documented across a wide range of SOAs (further arguing against a latent bottleneck), and (3) bypassing was observed for a very large percentage of participants. It is also impressive that bypassing was possible even though only one of the two tasks was highly practiced. This finding suggests that one task was automatized, rather than that the participants learned to integrate the two tasks in some way (for more discussion of this issue, see Ruthruff, Johnston, et al., 2006). This finding might not be universal, however. Oberauer and Kliegl (2004), for instance, found evidence that participants could perform two updates of working memory (a location and a number) in parallel after 24 practice sessions (see also Göthe, Oberauer, \& Kliegl, 2007), but only when both memory updates were practiced together. Further research is needed to determine what factors are responsible for the differences in findings.

Although we have concluded that the majority of the participants bypassed the bottleneck in Experiment 1, note that these participants did not eliminate dual-task interference completely. Hazeltine and Ruthruff (2006) have recently argued that, even when the bottleneck is bypassed, there may be other sources of central interference, such as competition between central codes of the tasks simultaneously residing in working memory (see also Hazeltine et al., 2006; Ruthruff, Hazeltine, \& Remington, 2006). Another possible source of dual-task costs is response grouping. If Task 2 finishes before Task 1 , the Task 2 response must be temporarily delayed in order to couple it with the Task 1 response.

When considering the likelihood of bottleneck bypass in the real world (e.g., driving while talking on a cell phone), it is important to note that the present tone task was very simple. There were only two tones (low pitched and high pitched), mapped compatibly onto two responses (low and high). It remains to be seen whether more complicated real-world tasks, with larger numbers of stimuli and responses, can also be automatized (see also Lien et al., 2006). If they can be automatized at all, it will probably require far greater numbers of trials than those used in the present experiments.

\section{Does an Automatized Task Greedily Recruit Available Central Resources?}

Having found clear evidence of bottleneck bypassing in Experiment 1, 5 of the 6 participants with the clearest evidence of having bypassed the bottleneck without response grouping (bypassers) were transferred to a new dual-task condition. We simply reversed the task order, so that the highly practiced auditory task served as Task 1 , instead of Task 2. If automatized tasks greedily recruit available central resources, the practiced Task 1 would engage central resources, and the less practiced Visual Task 2 should experience a bottleneck delay. Testing this hypothesis is tricky, because an automatized Task 1 will tend to produce fast responses and, therefore, will interfere little with Task 2 even if the central bottleneck is intact. To mitigate this problem, we gave Task 2 a $150-\mathrm{msec}$ head start, relative to the initial set of SOAs used in Experiment 1. This manipulation aimed to better temporally align the demand for the Task 1 and Task 2 central operations. 
Although the PRP effect remained small, it was larger than that observed in Experiment 1, even though mean RT1 was much shorter. We also found several other converging indicators that, when taken together, support the presence of a central bottleneck in Experiment 2. In particular, there was a linear relationship between the PRP effect and RT1, with a slope close to 1.0 across participants. Also, we observed dependencies between RT2 and RT1 at the shortest SOA. The last - and perhaps most compelling - piece of evidence for the presence of a central bottleneck in Experiment 2 stemmed from the near absence of response reversals at the -135 -msec SOA $(0.5 \%)$. We estimated that if there were no central bottleneck, allowing the tasks to be performed independently, response reversals should have occurred on $16.7 \%$ of the trials. Note that none of these indicators of a bottleneck were present in this sample of participants in Experiment 1. In summary, even the participants who were able to bypass the central bottleneck in Experiment 1 appeared to be subject to a central bottleneck in Experiment 2.

The greater prevalence of a bottleneck in Experiment 2 than in Experiment 1 lends some support to the greedy resource recruitment hypothesis, which says that an automatized Task 2 (in this case, the tone task) will tend to recruit available central resources even though it does not really need them. Although counterintuitive at first blush, the automatic recruitment of central resources might be an effective strategy. This reflexive assignment of central resources relieves the system from the need to assess whether the task actually needs those resources (a potentially timeconsuming and resource-consuming judgment). Although this allocation strategy may cause seemingly unnecessary delays in other tasks that arrive later, one can speculate that this allocation policy will usually produce little cost. In the present Experiment 2, for instance, dual-task costs occurred only at the shortest SOAs and were modest in size when they did occur.

Further research is needed to more thoroughly test the greedy resource recruitment hypothesis and whether it applies beyond the present tasks. Another interesting question is whether, with extended dual-task practice on a pair of tasks, the cognitive system might be able to somehow override the automatic assignment of central resources to an automatized Task 1 . Note that the participants received only three dual-task sessions in Experiment 2 and that the majority of trials used long SOAs, for which there should be no cost of greedy resource recruitment.

\section{Conclusions}

In summary, in a PRP paradigm that paired a highly practiced Task 2 with an unpracticed Task 1, we found evidence of bottleneck bypassing in the majority $(85 \%)$ of participants. These results represent an important exception to the seemingly ubiquitous inability of the brain to simultaneously select two independent actions. Furthermore, additional dual-task transfer sessions with participants previously identified as bypassers provided evidence of a central bottleneck when the highly practiced task came first. These results suggest that automatized tasks, which do not need central resources, tend to greed- ily recruit central resources when available, making them unavailable to other tasks.

\section{AUTHOR NOTE}

This research was supported by a postdoctoral fellowship from La Fondation des Gouverneurs of the Institut Universitaire de Gériatrie de Montréal to F.M. This research was also supported by a Canadian grant from the Natural Sciences and Engineering Council of Canada and by salary support from the Fonds de Recherche en Santé du Québec to L.B. We thank our participants for their diligence. We are also grateful for the assistance of Julie Brunet, Christine Gagnon, Liliane Issa, and Jocelyn Bélanger in carrying out this research project. We also thank Klaus Oberauer, Michael Tombu, and two anonymous reviewers for useful comments on earlier versions of the manuscript. In addition, we thank Alan Hartley for his commentary and insights. Correspondence concerning this article should be addressed to F. Maquestiaux, UFR STAPS de 1'Université Paris-Sud, JE 2494, Bâtiment 335, 91405 Orsay Cedex, France; or to E. Ruthruff, Department of Psychology, Logan Hall, University of New Mexico, Albuquerque, NM 87131-1161 (e-mail: francois .maquestiaux@u-psud.fr or ruthruff@unm.edu).

\section{REFERENCES}

Anderson, J. R., TAatgen, N. A., \& Byrne, M. D. (2005). Learning to achieve perfect timesharing: Architectural implications of Hazeltine, Teague, and Ivry (2002). Journal of Experimental Psychology: Human Perception \& Performance, 31, 749-761.

Bertelson, P., \& Tisseyre, F. (1969). Refractory period of c-reactions. Journal of Experimental Psychology, 79, 122-128.

BORGER, R. (1963). The refractory period and serial choice reactions. Quarterly Journal of Experimental Psychology, 15, 1-12.

DE Jong, R. (1993). Multiple bottlenecks in overlapping task performance. Journal of Experimental Psychology: Human Perception \& Performance, 19, 965-989.

Göthe, K., Oberauer, K., \& Kliegl, R. (2007). Age differences in dual-task performance after practice. Psychology \& Aging, 22, 596-606.

Hartley, A. A., \& Maquestiaux, F. (2007). Success and failure at dual-task coordination by younger and older adults. Psychology \& Aging, 22, 215-222.

Hazeltine, E., \& Ruthruff, E. (2006). Modality pairing effects and the response selection bottleneck. Psychological Research, 70, 504-513.

Hazeltine, E., Ruthruff, E., \& Remington, R. W. (2006). The role of input and output modality pairings in dual-task performance: Evidence for content-dependent central interference. Cognitive Psychology, 52, 291-345.

HaZeltine, E., Teague, D., \& Ivry, B. (2002). Simultaneous dual-task performance reveals parallel response selection after practice. Journal of Experimental Psychology: Human Perception \& Performance, 28, 527-545.

Karlin, L., \& Kestenbaum, R. (1968). Effects of number of alternatives on the psychological refractory period. Quarterly Journal of Experimental Psychology, 20, 167-178.

Keele, S. (1973). Attention and human performance. Pacific Palisades, CA: Goodyear.

LeVy, J., \& PAshler, H. (2001). Is dual-task slowing instruction dependent? Journal of Experimental Psychology: Human Perception \& Performance, 27, 862-869.

Levy, J., Pashler, H., \& Boer, E. (2006). Central interference in driving: Is there any stopping the psychological refractory period? Psychological Science, 17, 228-235.

Lien, M.-C., McCann, R. S., Ruthruff, E., \& Proctor, R. W. (2005). Dual-task performance with ideomotor-compatible tasks: Is the central processing bottleneck intact, bypassed, or shifted in locus? Journal of Experimental Psychology: Human Perception \& Performance, 31, 122-144.

Lien, M.-C., \& Proctor, R. W. (2002). Stimulus-response compatibility and psychological refractory period effects: Implications for response selection. Psychonomic Bulletin \& Review, 9, 212-238.

Lien, M.-C., RuthrufF, E., \& Johnston, J. C. (2006). Attentional limi- 
tations in doing two things at once: The search for exceptions. Current Directions in Psychological Science, 15, 89-93.

Maquestiaux, F., Hartley, A. A., \& Bertsch, J. (2004). Can practice overcome age-related differences in the psychological refractory period effect? Psychology \& Aging, 19, 649-667.

McCann, R. S., \& Johnston, J. C. (1992). Locus of the single-channel bottleneck in dual-task interference. Journal of Experimental Psychology: Human Perception \& Performance, 18, 471-484.

Meyer, D. E., \& Kieras, D. E. (1997a). A computational theory of executive cognitive processes and multiple-task performance: Part 1. Basic mechanisms. Psychological Review, 104, 3-65.

Meyer, D. E., \& Kieras, D. E. (1997b). A computational theory of executive cognitive processes and multiple-task performance: Part 2. Accounts of psychological refractory-period phenomena. Psychological Review, 104, 749-791.

Oberauer, K., \& Kliegl, R. (2004). Simultaneous cognitive operations in working memory after dual-task practice. Journal of Experimental Psychology: Human Perception \& Performance, 30, 689-707.

Pashler, H. [E.] (1994a). Dual-task interference in simple tasks: Data and theory. Psychological Bulletin, 116, 220-244.

PASHLER, H. [E.] (1994b). Graded capacity-sharing in dual-task interference? Journal of Experimental Psychology: Human Perception \& Performance, 20, 330-342.

Pashler, H. E. (1998). The psychology of attention. Cambridge, MA: MIT Press.

Pashler, H. [E.], \& Johnston, J. C. (1989). Chronometric evidence for central postponement in temporally overlapping tasks. Quarterly Journal of Experimental Psychology, 41A, 19-45.

Ruthruff, E., Hazeltine, E., \& Remington, R. W. (2006). What causes residual dual-task interference after practice? Psychological Research, 70, 494-503.

Ruthruff, E., Johnston, J. C., \& VAn Selst, M. (2001). Why practice reduces dual-task interference. Journal of Experimental Psychology: Human Perception \& Performance, 27, 3-21.

Ruthruff, E., Johnston, J. C., Van Selst, M., Whitsell, S., \& RemINGTON, R. (2003). Vanishing dual-task interference after practice: Has the bottleneck been eliminated or is it merely latent? Journal of Experimental Psychology: Human Perception \& Performance, 29, 280-289.

Ruthruff, E., Pashler, H. E., \& Hazeltine, E. (2003). Dual-task interference with equal task emphasis: Graded capacity sharing or central postponement? Perception \& Psychophysics, 65, 801-816.

Ruthruff, E., Pashler, H. E., \& Klaassen, A. (2001). Processing bottlenecks in dual-task performance: Structural limitation or strategic postponement? Psychonomic Bulletin \& Review, 8, 73-80.

Ruthruff, E., Van Selst, M., Johnston, J. C., \& Remington, R. W. (2006). How does practice reduce dual-task interference: Integration, automatization, or just stage-shortening? Psychological Research, 70, $125-142$.

Schumacher, E. H., Lauber, E. J., Glass, J. M., Zurbriggen, E. L., Gmeindl, L., Kieras, D. E., \& Meyer, D. E. (1999). Concurrent response-selection processes in dual-task performance: Evidence for adaptive executive control of task scheduling. Journal of Experimental Psychology: Human Perception \& Performance, 25, 791-814.

Schumacher, E. H., Seymour, T. L., Glass, J. M., Fencsik, D. E., Lauber, E. J., Kieras, D. E., \& Meyer, D. E. (2001). Virtually perfect time sharing in dual-task performance: Uncorking the central cognitive bottleneck. Psychological Science, 12, 101-108.

Stelzel, C., Schumacher, E. H., Schubert, E. H., \& D'Esposito, M. (2006). The neural effect of stimulus-response modality compatibility on dual-task performance: An fMRI study. Psychological Research, 70, 514-525.

Strayer, D. L., Drews, F. A., \& Johnston, W. A. (2003). Cell phoneinduced failures of visual attention during simulated driving. Journal of Experimental Psychology: Applied, 9, 23-32.

Telford, C. W. (1931). Refractory phase of voluntary and associative responses. Journal of Experimental Psychology, 14, 1-35.

Tombu, M., \& Joliceur, P. (2004). Virtually no evidence for virtually perfect time-sharing. Journal of Experimental Psychology: Human Perception \& Performance, 30, 795-810.

Van Selst, M., Ruthruff, E., \& Johnston, J. C. (1999). Can practice eliminate the psychological refractory period effect? Journal of Experimental Psychology: Human Perception \& Performance, 25, 1268-1283.

Welford, A. T. (1952). The "psychological refractory period" and the timing of high-speed performance: A review and a theory. British Journal of Psychology, 43, 2-19.

\section{NOTES}

1. This simplified equation assumes that the short SOA is sufficiently short that the bottleneck is encountered on every trial and that the long SOA is sufficiently long that the bottleneck is never encountered. For a more general formula, see Ruthruff, Johnston, and Van Selst (2001).

2. As has been explained below, these participants often emitted both responses as a couplet at the shortest SOA. This marked tendency to group responses very likely contributed to the somewhat lower incidence of response reversals at the short SOAs, relative to the participants with the smallest PRP effects. The reason is that when Task 1 and Task 2 responses are grouped and emitted as a couplet, response reversals should occur on only half of the trials. Also, a close examination of Table 1 indicates that the Task 1 error rate further increased from the longest to the shortest SOA in these 11 participants $(5.8 \%)$, relative to the 6 participants with small PRPs (1.1\%) and the 3 participants with large PRPs (1.5\%). This $5.8 \%$ Task 1 error rate increase with decreasing SOAs, very likely caused by the grouping of Task 1 and Task 2 responses at short SOAs, might be responsible for the overall 3.8\% Task 1 error rate increase from the longest to the shortest SOA observed in the overall data from all 20 participants.

\section{APPENDIX \\ Estimating the Amount of Task 1 Slowing Predicted by a Central Bottleneck With the Reversed Central-Processing Order}

Here, we describe the procedures used to estimate the amount of Task 1 slowing that should have occurred in Experiment 1, assuming a central bottleneck with Task 2 central operations often performed before Task 1 central operations. These estimations were conducted separately for the suspected bypassers and suspected bypass groupers. Having produced these estimates, they can then be compared against the actual amount of observed Task 1 slowing, which was negligible for each of these two groups.

The first step is to determine the proportion of trials on which RT1 lengthening should have occurred. We calculated this proportion as the number of trials with a reversed response order, plus any additional response grouping trials with an IRI $<100 \mathrm{msec}$. Response grouping trials can arise from either the reversed or the regular central-processing order; but even with the normal processing order, RT1 would still be delayed because it must wait for the second response to be prepared. In some previous studies, a criterion of $150 \mathrm{msec}$ has been used for identifying response grouping trials, but we wanted to make sure that our estimates were conservative. With this approach, the proportion of trials producing RT1 lengthening at the 15-, 65-, and 150-msec SOAs was $97.9 \%$, $95.0 \%$, and $85.2 \%$, respectively, for the bypassers and $80.9 \%, 81.3 \%$, and $71.4 \%$ for the bypass groupers.

The next step is to use related experiments concluding in favor of a bottleneck to infer how much PRP effect would be expected in the present Experiment 1. Fortunately, there are two such related experiments, using 


\section{APPENDIX (Continued)}

tasks identical (or nearly identical) to those in Experiment 1. The first case is the present Experiment 2, which was completed by 5 of the 6 bypassers identified in Experiment 1. The task order was reversed, relative to Experiment 1 , and the data provided evidence of a processing bottleneck. Critically, the central-processing order believed to have occurred in Experiment 2 (tone task before visual task) is exactly the order considered as the explanation for Experiment 1. Thus, this experiment provides an ideal basis for comparison.

The short SOAs in Experiment $2(-135,-85$, and $0 \mathrm{msec})$ do not perfectly align with those of Experiment 1 $(15,65$, and $150 \mathrm{msec})$, but they are very close (within $20 \mathrm{msec})$. Note that the difference in sign simply reflects the fact that SOA is defined as the time between S1 and S2, which corresponded to different tasks in the two experiments; the event sequences were actually nearly identical.

As an approximation, one could simply estimate the amount of Task 1 slowing in Experiment 1 on the basis of the PRP effect observed for Task 2 in Experiment 2. However, it is important to acknowledge that the tone task was performed more quickly in Experiment 2 (due to greater practice and the switch in emphasis from the visual task to the tone task). To take this factor into account, we calculated the function relating the PRP effect and RT1 across participants at each of the three shortest SOAs in Experiment 2 (i.e., those matching the three shortest SOAs in Experiment 1). We then plugged in the Experiment 1 value for "RT1" (which actually was RT2, since Task 2 is hypothesized to have been performed first). In Experiment 2, the PRP:RT1 functions were the following: $\mathrm{PRP}^{\prime}=.257(\mathrm{RT} 1)-34.549, r^{2}=.075$ at the $0-\mathrm{msec} \mathrm{SOA}$; PRP' $=.793(\mathrm{RT} 1)-167.180, r^{2}=$ .413 at the $-85-\mathrm{msec} \mathrm{SOA}$; and $\mathrm{PRP}^{\prime}=.839(\mathrm{RT} 1)-181.814, r^{2}=.351$ at the $-135-\mathrm{msec}$ SOA.

To corroborate the estimates based on the present Experiment 2, we also used PRP findings from Van Selst et al. (1999). They adopted tasks very similar to those used in Experiment 1, but presented in an order opposite to that in our Experiment 1 (the same order as that used in our Experiment 2), and concluded in favor of a processing bottleneck. Also, their short SOA (17 msec) was nearly identical to ours (15 msec). Thus, although their participants were, of course, different from ours, their study can provide a useful basis for comparison. Their design included only dual-task trials, which allowed them to track the decline in the PRP effect across sessions. It turns out that the PRP effect declined roughly millisecond for millisecond with the decline in mean RT1. The equation they derived was $\mathrm{PRP}^{\prime}=1.022(\mathrm{RT} 1)-257.342\left(r^{2}=.959\right)$. We applied this equation to the suspected bypassers in the present Experiment 1, inserting the Experiment 1 "RT1" value (which, again, came from the observed mean RT2).

The final step was to multiply the estimated amount of Task 1 slowing when the central-processing order was reversed by the probability that the central-processing order was reversed. The results are described in the main text. 